\title{
Localizing energy sources and sinks in plates using power flow maps computed from laser vibrometer measurements ${ }^{1}$
}

\author{
J.R.F. Arruda ${ }^{\mathrm{a}, *}$ and P. Mas ${ }^{\mathrm{b}, 2}$ \\ ${ }^{a}$ Departamento de Mecânica Computacional, \\ Universidade Estadual de Campinas, Cidade \\ Universitária "Zeferino Vaz,, \\ FEM-UNICAMP-Caixa Postal 6122, \\ CEP 13083-970 Campinas, SP, Brazil \\ ${ }^{\mathrm{b}}$ Department of Mechanical Engineering, Katholieke \\ Universiteit Leuven, Leuven, Belgium \\ Received December 1997 \\ Revised 31 August 1998
}

This paper presents an experimental method especially adapted for the computation of structural power flow using spatially dense vibration data measured with scanning laser Doppler vibrometers. In the proposed method, the operational deflection shapes measured over the surface of the structure are curve-fitted using a two-dimensional discrete Fourier series approximation that minimizes the effects of spatial leakage. From the wavenumber-frequency domain data thus obtained, the spatial derivatives that are necessary to determine the structural power flow are easily computed. Divergence plots are then obtained from the computed intensity fields. An example consisting of a rectangular aluminum plate supported by rubber mounts and excited by a point force is used to appraise the proposed method. The proposed method is compared with more traditional finite difference methods. The proposed method was the only to allow the localization of the energy source and sinks from the experimental divergence plots.

Keywords: Power flow, structural intensity, plates, energy flow, energy sinks, laser vibrometer

\footnotetext{
${ }^{1}$ Editor's note: This article was presented in an earlier form at the International Conference on Vibration Measurements by Laser Techniques, Anacona, Italy. The conference was organized by the Italian Association of Laser Velocimetry. The articles were selected, edited, and reviewed by a committee chaired by Professor Enrico Primo Tomasini, Department of Mechanics, Faculty of Engineering, University of Ancona, Italy.
}

\section{Introduction}

Predicting and measuring elastic waves propagating through a structure can be of foremost importance in vibroacoustic problems. The prediction and the measurement of the propagating elastic waves within a structure is usually referred to as structural power flow, vibration intensity, or structural intensity, the latter denominations coming from the analogy with acoustic intensity. The power flow to localized dampers or to neighboring structures and supports can be cardinal mechanisms through which structural vibration is damped out, which partly explains the practical difficulty is estimating internal damping coefficients in structures from ground vibration tests. It can also be a key for solving structure borne noise problems, by channeling vibrations to where they do not radiate noise, instead of trying to suppress them.

Power flow refers to the active part of the vibration energy. As the active energy is usually only a small fraction of the total vibration energy, estimating it from measured vibration is not simple. Measuring vibration intensity is more elaborate than measuring acoustic intensity. Sound propagates through compression-type waves only, while vibration propagates through two basic types of waves: compression and shear waves. Depending on the geometry of the continuum, these two basic types of waves combine into different types of waves, such as bending, torsional, and longitudinal waves, which must be measured. In the case of beams and plates, bending waves play a major role, for they are usually responsible for most of the sound radiated from the structure surface.

Similarly to acoustic intensity, vibration intensity measurements are also associated with cross mea-

\footnotetext{
*Corresponding author. Tel.: +55 197883386 or 788 7540; fax: +55 19289 3722; e-mail: arruda@fem.unicamp.br.

${ }^{2}$ Presently working with Leuven Measurement Systems Engineering, Leuven, Belgium.
} 
surements between closely-spaced transducers, which are microphones in the former case and, usually, accelerometers in the latter. Following the original work by Noiseaux [8], many authors have investigated different ways of computing the bending vibration intensity from measured accelerations in beams and plates (see, for instance $[4,7,11,13]$ ). Most authors use finitedifference approximations to compute the partial spatial derivatives that are necessary to compute the power flow.

In the case of flexural power flow in beams, an analytical solution, valid for any homogeneous span without discontinuities, external forces, or boundary conditions, exists. Therefore, the assumption of far field is not mandatory, and the near field effects can, in theory, be taken into account when computing the power flow from measurements. If the theoretical dispersion relation (wave number as a function of frequency) is used, four adjacent acceleration measurements, for instance, are sufficient to determine the power flow. If the dispersion relation is to be estimated, a minimum of five measurements are needed. However, Bauman [2] has recently compared the existing finite-difference based methods, and concluded that none succeed in adequately estimating the nearfield effects.

In the case of plates, a complete solution is not available when there are boundaries with energy losses [8], which are always present in power flow problems. Therefore, an assumption of superposition of plane waves propagating in different directions is usually made. Furthermore, it is generally assumed that these waves do not interact (free field). Thus, it becomes possible to compute the power flow with a finite difference approximation using as few as 3 measurements. If one wants to obtain the wave numbers as well, in two orthogonal directions, 5 measurements are necessary.

More recent papers investigate the computation of the power flow in plates without using the finitedifferences approximation. Pascal et al. $[9,10]$ have proposed the use of spatial Fourier transforms to compute the power flow in the wavenumber domain. The major advantage is the fact that it is not necessary to make simplifying assumptions concerning the flexural vibration field.

In this text, the basic formulation for the structural power flow in plates and the methods for computing it from measured accelerations using finite difference approximations will be reviewed. A method using a twodimensional Fourier series approximation is proposed.
The use of a two-dimensional discrete Fourier series (DFT) interpolation in structural power flow computations is not new [5], but its use as an approximation to allow non-equally spaced measurements and to avoid leakage problems seem to be novel. The approximation is computed using the Regressive Discrete Fourier Series (RDFS) previously developed by one of the authors [1].

An example of a rectangular plate with a point force and localized dampers will be used to illustrate the above methods. Both numerical simulation and experimental results are shown.

\section{Basic relations}

In this section, the basic theory of flexural power flow in thin homogeneous flat plates is reviewed, so that a coherent set of equations can be derived. The formulae are well established in the literature, but there are usually minor differences in the definitions used by different authors, which nevertheless make the comparison between measured and computed quantities difficult. The classical plate theory, which is the equivalent in two dimensions of the Bernoulli-Euler beam theory, is used here. Rotational inertia and shear deformations are neglected. In the low frequency range, encompassing the first few tens of modes, these hypotheses are reasonable.

The bending-wave equation in the classical plate theory may be written as [5]:

$$
\begin{aligned}
D & \nabla^{2} \nabla^{2} w(x, y, t)+\rho h \frac{\partial^{2} w(x, y, t)}{\partial t^{2}} \\
& =F(t) \delta\left(x_{0}, y_{0}\right)
\end{aligned}
$$

where $D=E h^{3} /\left(12\left(1-v^{2}\right)\right)$ is the flexural stiffness, $E$ is the Young's modulus, $v$ is the Poisson's ratio, $h$ is the plate thickness, $w(x, y, t)$ is the transverse displacement, $\rho$ is the mass density, $F(t) \delta\left(x_{0}, y_{0}\right)$ is a point external force, and $\nabla^{2}=\partial^{2} / \partial x^{2}+\partial^{2} / \partial y^{2}$ (Laplacian). Assuming a solution of the type:

$$
w(x, y, t)=\Re\left\{W(x, y, \omega) \mathrm{e}^{\mathrm{i} \omega t}\right\},
$$

where

$W(x, y, \omega)=\sum_{m} V_{m} \exp \left(-\mathrm{i} k\left(x \cos \alpha_{m}+y \sin \alpha_{m}\right)\right)$, 
and $k^{4}=\omega^{2} \rho h / D$, with $\Re$ denoting the real part of a complex quantity and $\mathrm{i}=\sqrt{-1}$, it is possible to write:

$$
\begin{aligned}
& \nabla^{2} w=-k^{2} w, \\
& \frac{\partial^{2} w}{\partial x^{2}}=-k_{x}^{2} w, \\
& \frac{\partial^{2} w}{\partial y^{2}}=-k_{y}^{2} w,
\end{aligned}
$$

where $k_{x}=k \cos (\alpha)$ and $k_{y}=k \sin (\alpha)$ are the dispersion relations in the orthogonal directions. Note that $k^{2}=k_{x}^{2}+k_{y}^{2}$.

Equation (1) is derived from the equilibrium equations of a flat plate element, which can be expressed as [5]:

$$
\begin{aligned}
& \frac{\partial Q_{x}}{\partial x}+\frac{\partial Q_{y}}{\partial y}=\rho h \frac{\partial^{2} w}{\partial t^{2}}-F(t), \\
& \frac{\partial M_{x}}{\partial x}+\frac{\partial M_{x y}}{\partial y}+Q_{x}=0, \\
& \frac{\partial M_{y}}{\partial y}+\frac{\partial M_{y x}}{\partial x}+Q_{y}=0 .
\end{aligned}
$$

The moments and shear forces may be expressed in terms of the displacement $w(x, y, t)$ as:

$$
\begin{aligned}
M_{x} & =D\left(\frac{\partial^{2} w}{\partial x^{2}}+v \frac{\partial^{2} w}{\partial y^{2}}\right), \\
M_{y} & =D\left(\frac{\partial^{2} w}{\partial y^{2}}+v \frac{\partial^{2} w}{\partial x^{2}}\right), \\
M_{x y} & =M_{y x}=D(1-v) \frac{\partial^{2} w}{\partial x \partial y}, \\
Q_{x} & =D \frac{\partial}{\partial x}\left(\nabla^{2} w\right), \\
Q_{y} & =D \frac{\partial}{\partial y}\left(\nabla^{2} w\right) .
\end{aligned}
$$

Substituting Eqs (6) and (7) into Eqs (5), Eq. (1) can be obtained.

The power flow along the plate in each orthogonal direction, $x$ and $y$, has three components:

$$
\begin{aligned}
& P_{x}(x, y)=\left\langle Q_{x} \dot{w}\right\rangle_{T}-\left\langle M_{x y} \dot{\theta}_{x}\right\rangle_{T}+\left\langle M_{x} \dot{\theta}_{y}\right\rangle_{T}, \\
& P_{y}(x, y)=\left\langle Q_{y} \dot{w}\right\rangle_{T}+\left\langle M_{y x} \dot{\theta}_{y}\right\rangle_{T}-\left\langle M_{y} \dot{\theta}_{x}\right\rangle_{T},
\end{aligned}
$$

where \langle\rangle$_{T}$ denotes the time average, and:

$$
\theta_{x}=\frac{\partial w}{\partial y}, \theta_{y}=-\frac{\partial w}{\partial x}, \dot{\theta}=\frac{\partial \theta}{\partial t}
$$

Substituting Eqs (6), (7) and (9) in Eqs (8) gives the following expression for the power flow per unit length in plates:

$$
\begin{aligned}
P_{x}(x, y)= & D\left\langle\frac{\partial}{\partial x}\left(\nabla^{2} w\right) \frac{\partial w}{\partial t}\right. \\
& -\left(\frac{\partial^{2} w}{\partial x^{2}}+v \frac{\partial^{2} w}{\partial y^{2}}\right) \frac{\partial^{2} w}{\partial x \partial t} \\
& \left.-(1-v) \frac{\partial^{2} w}{\partial x \partial y} \frac{\partial^{2} w}{\partial y \partial t}\right\rangle_{T} \\
P_{y}(x, y)= & D\left\langle\frac{\partial}{\partial y}\left(\nabla^{2} w\right) \frac{\partial w}{\partial t}\right. \\
& -\left(\frac{\partial^{2} w}{\partial y^{2}}+v \frac{\partial^{2} w}{\partial x^{2}}\right) \frac{\partial^{2} w}{\partial y \partial t} \\
& \left.-(1-v) \frac{\partial^{2} w}{\partial x \partial y} \frac{\partial^{2} w}{\partial x \partial t}\right\rangle_{T} .
\end{aligned}
$$

Assuming harmonic vibration and the wave superposition of Eq. (3), one can formulate the time-average power flow for each of the three components separately. For this purpose we use Eqs (4) and the relation $\partial w / \partial t=\mathrm{i} \omega w$.

- Bending:

Expressing the power flow in terms of accelerations $\ddot{w}$ :

$$
\begin{aligned}
& P_{x}^{b}(x, y)=D\left(k_{x}^{2}+v k_{y}^{2}\right)\left\langle\frac{1}{\omega^{3}} \ddot{w}\left(\mathrm{i} \frac{\partial \ddot{w}}{\partial x}\right)\right\rangle_{T}, \\
& P_{y}^{b}(x, y)=D\left(k_{y}^{2}+v k_{x}^{2}\right)\left\langle\frac{1}{\omega^{3}} \ddot{w}\left(\mathrm{i} \frac{\partial \ddot{w}}{\partial y}\right)\right\rangle_{T}
\end{aligned}
$$

- Shear:

$$
\begin{aligned}
& P_{x}^{S}(x, y)=-D\left(k_{x}^{2}+k_{y}^{2}\right)\left\langle\frac{1}{\omega^{3}} \frac{\partial \ddot{w}}{\partial x}(\mathrm{i} \ddot{w})\right\rangle_{T}, \\
& P_{y}^{S}(x, y)=-D\left(k_{x}^{2}+k_{y}^{2}\right)\left\langle\frac{1}{\omega^{3}} \frac{\partial \ddot{w}}{\partial y}(\mathrm{i} \ddot{w})\right\rangle_{T} .
\end{aligned}
$$

- Torsion: 


$$
\begin{aligned}
& P_{x}^{t}(x, y)=-D(1-v)\left\langle\frac{1}{\omega^{3}} \frac{\partial^{2} \ddot{w}}{\partial x \partial y}\left(\mathrm{i} \frac{\partial \ddot{w}}{\partial y}\right)\right\rangle_{T}, \\
& P_{y}^{t}(x, y)=-D(1-v)\left\langle\frac{1}{\omega^{3}} \frac{\partial^{2} \ddot{w}}{\partial y \partial x}\left(\mathrm{i} \frac{\partial \ddot{w}}{\partial x}\right)\right\rangle_{T} .
\end{aligned}
$$

- Note that it is important to keep the $i$ next to the term to which it is associated, as this will yield the correct sign when taking the complex conjugate.

Some further assumptions can be made regarding the torsion component in order to obtain a simpler formulation. There are different ways of doing this, which lead to different expressions for the power flow, when associated with some form of approximation of the spatial derivatives by finite differences.

The most commonly used assumption is the non interaction of the superposed waves. Thus, the out-ofplane displacements $w(x, y, t)$ can be expressed by a single two-dimensional wave:

$$
\begin{aligned}
w(x, y, t)= & \left(A_{1} \mathrm{e}^{-\mathrm{i} k_{x} x}+B_{1} \mathrm{e}^{\mathrm{i} k_{x} x}\right) \\
& \times\left(A_{2} \mathrm{e}^{-\mathrm{i} k_{y} y}+B_{2} \mathrm{e}^{\mathrm{i} k_{y} y}\right) \mathrm{e}^{\mathrm{i} \omega t}
\end{aligned}
$$

and it is straightforward to show that:

$$
\begin{aligned}
& \frac{\partial^{2} w}{\partial x \partial y} \frac{\partial^{2} w}{\partial y \partial t}=-k_{y}^{2}\left(\frac{\partial w}{\partial x} \frac{\partial w}{\partial t}\right)+\mathrm{cst} \\
& \frac{\partial^{2} w}{\partial x \partial y} \frac{\partial^{2} w}{\partial x \partial t}=-k_{x}^{2}\left(\frac{\partial w}{\partial y} \frac{\partial w}{\partial t}\right)+\text { cst. }
\end{aligned}
$$

The constant term is non-propagating, and, therefore, can be neglected in the power flow expression. Instead of using this relation, Pascal et al. $[9,10]$ used the assumption that there is no interaction between waves, i.e., $V_{n} V_{m}^{*}=0$ if $n \neq m$, in which case it can be shown that $\nabla \times\left(w \nabla w^{*}\right)=0$. With this assumption, Eqs (13) may be rewritten as:

$$
\begin{aligned}
& P_{x}^{t}(x, y)=D(1-v) k_{y}^{2}\left\langle\frac{1}{\omega^{3}} \frac{\partial \ddot{w}}{\partial x}(\mathrm{i} \ddot{w})\right\rangle_{T}, \\
& P_{y}^{t}(x, y)=D(1-v) k_{x}^{2}\left\langle\frac{1}{\omega^{3}} \frac{\partial \ddot{w}}{\partial y}(\mathrm{i} \ddot{w})\right\rangle_{T}
\end{aligned}
$$

The relation:

$$
\langle x(t) y(t)\rangle_{T}=\frac{1}{2} \Re\left\{X(\omega) Y(\omega)^{*}\right\},
$$

which can be applied to sinusoidal signals, as well as to periodic, transient, or random signals may be used to obtain frequency-domain formulas. It should be noted that $X(\omega)$ is the Fourier transform, series, discrete transform, or finite transform of $x(t)$.

Using this relation, it is possible to write:

$$
\begin{aligned}
& P_{x}^{b}(x, y)=D\left(k_{x}^{2}+v k_{y}^{2}\right) \frac{1}{2} \Re\left\{\frac{1}{\omega^{3}} \ddot{W}\left(\mathrm{i} \frac{\partial \ddot{W}}{\partial x}\right)^{*}\right\}, \\
& P_{y}^{b}(x, y)=D\left(k_{y}^{2}+v k_{x}^{2}\right) \frac{1}{2} \Re\left\{\frac{1}{\omega^{3}} \ddot{W}\left(\mathrm{i} \frac{\partial \ddot{W}}{\partial y}\right)^{*}\right\}, \\
& P_{x}^{S}(x, y)=-D\left(k_{x}^{2}+k_{y}^{2}\right) \frac{1}{2} \Re\left\{\frac{1}{\omega^{3}} \frac{\partial \ddot{W}}{\partial x}(\mathrm{i} \ddot{W})^{*}\right\}, \\
& P_{y}^{S}(x, y)=-D\left(k_{x}^{2}+k_{y}^{2}\right) \frac{1}{2} \Re\left\{\frac{1}{\omega^{3}} \frac{\partial \ddot{W}}{\partial y}(\mathrm{i} \ddot{W})^{*}\right\}, \\
& P_{x}^{t}(x, y)=D(1-v) k_{y}^{2} \frac{1}{2} \Re\left\{\frac{1}{\omega^{3}} \frac{\partial \ddot{W}}{\partial x}(\mathrm{i} \ddot{W})^{*}\right\}, \\
& P_{y}^{t}(x, y)=D(1-v) k_{x}^{2} \frac{1}{2} \Re\left\{\frac{1}{\omega^{3}} \frac{\partial \ddot{W}}{\partial y}(\mathrm{i} \ddot{W})^{*}\right\} .
\end{aligned}
$$

As $\Re\{\mathrm{i}()\}=-\Im\{()\}$ and $\Im\left(A B^{*}\right)=-\Im\left(A^{*} B\right)$, it is possible to add the three components and obtain a simple expression for the total power flow in the two directions:

$$
\begin{aligned}
& P_{x}(x, y)=\frac{D\left(k_{x}^{2}+v k_{y}^{2}\right)}{\omega^{3}} \Im\left\{\ddot{W} \frac{\partial \ddot{W}^{*}}{\partial x}\right\}, \\
& P_{y}(x, y)=\frac{D\left(k_{y}^{2}+v k_{x}^{2}\right)}{\omega^{3}} \Im\left\{\ddot{W} \frac{\partial \ddot{W}^{*}}{\partial y}\right\} .
\end{aligned}
$$

This formulation, due to Meyer and Thomasson [7], is not the most suitable for computing the power flow, as it involves the wavenumbers in the orthogonal directions, $k_{x}$ and $k_{y}$, which, unlike $k$, do not have an analytical expression, as they depend on the propagation direction. Therefore, to be able to use Eq. (19), one has to determine first $k_{x}$ and $k_{y}$. Another formulation, due to Pascal et al. [9,10], yields a slightly different result which is more suitable for practical applications. Starting from Eqs (10), and using the relation given by Eq. (17), it is possible to write:

$$
\begin{aligned}
P_{x}(x, y)= & \frac{D}{2} \Re\left\{\frac{\partial}{\partial x}\left(\nabla^{2} W\right) \frac{\partial W^{*}}{\partial t}\right. \\
& -\left(\frac{\partial^{2} W}{\partial x^{2}}+v \frac{\partial^{2} W}{\partial y^{2}}\right) \frac{\partial^{2} W}{\partial x \partial t}
\end{aligned}
$$




$$
\begin{aligned}
& \left.-(1-v) \frac{\partial^{2} W}{\partial x \partial y} \frac{\partial^{2} W^{*}}{\partial y \partial t}\right\}, \\
& P_{y}(x, y)=\frac{D}{2} \Re\left\{\frac{\partial}{\partial y}\left(\nabla^{2} W\right) \frac{\partial W^{*}}{\partial t}\right. \\
& -\left(\frac{\partial^{2} W}{\partial y^{2}}+v \frac{\partial^{2} W}{\partial x^{2}}\right) \frac{\partial^{2} W^{*}}{\partial y \partial t} \\
& \left.-(1-v) \frac{\partial^{2} W}{\partial x \partial y} \frac{\partial^{2} W^{*}}{\partial x \partial t}\right\} \text {. }
\end{aligned}
$$

Substituting $\partial W / \partial t=\dot{W}$ and $W=-\mathrm{i} \dot{W} / \omega$ in the previous equation yields:

$$
\begin{aligned}
P_{x}(x, y)= & \frac{D}{2 \omega} \Im\left\{\frac{\partial}{\partial x}\left(\nabla^{2} \dot{W}\right) \dot{W}^{*}\right. \\
& -\left(\frac{\partial^{2} \dot{W}}{\partial x^{2}}+v \frac{\partial^{2} \dot{W}}{\partial y^{2}}\right) \frac{\partial^{2} \dot{W}^{*}}{\partial x} \\
& \left.-(1-v) \frac{\partial^{2} \dot{W}}{\partial x \partial y} \frac{\partial^{2} \dot{W}^{*}}{\partial y}\right\}, \\
P_{y}(x, y)= & \frac{D}{2 \omega} \Im\left\{\frac{\partial}{\partial y}\left(\nabla^{2} \dot{W}\right) \dot{W}^{*}\right. \\
& -\left(\frac{\partial^{2} \dot{W}}{\partial y^{2}}+v \frac{\partial^{2} \dot{W}^{2}}{\partial x^{2}}\right) \frac{\partial^{2} \dot{W}^{*}}{\partial y} \\
& \left.-(1-v) \frac{\partial^{2} \dot{W}}{\partial x \partial y} \frac{\partial^{2} \dot{W}^{*}}{\partial x}\right\} .
\end{aligned}
$$

Now adding to Eq. (21) the null term

$$
\left(\frac{\partial^{2} \dot{W}}{\partial y^{2}}-\frac{\partial^{2} \dot{W}}{\partial y^{2}}\right) \frac{\partial \dot{W}^{*}}{\partial y}
$$

and rearranging gives:

$$
\begin{aligned}
P_{x}(x, y)= & \frac{D}{2 \omega} \Im\left\{\frac{\partial}{\partial x}\left(\nabla^{2} \dot{W}\right) \dot{W}^{*}-\nabla^{2} \dot{W} \frac{\partial \dot{W}^{*}}{\partial x}\right. \\
& \left.-(1-v)\left(\frac{\partial^{2} \dot{W}}{\partial x \partial y} \frac{\partial \dot{W}^{*}}{\partial y}-\frac{\partial^{2} \dot{W}}{\partial y^{2}} \frac{\partial \dot{W}^{*}}{\partial x}\right)\right\}, \\
P_{y}(x, y)= & \frac{D}{2 \omega} \Im\left\{\frac{\partial}{\partial y}\left(\nabla^{2} \dot{W}\right) \dot{W}^{*}-\nabla^{2} \dot{W} \frac{\partial \dot{W}^{*}}{\partial y}\right. \\
& \left.-(1-v)\left(\frac{\partial^{2} \dot{W}}{\partial x \partial y} \frac{\partial \dot{W}^{*}}{\partial x}-\frac{\partial^{2} \dot{W}}{\partial x^{2}} \frac{\partial \dot{W}^{*}}{\partial y}\right)\right\} .
\end{aligned}
$$

The last terms in the above expression may be shown to be the $x$ and $y$ components of $\frac{1}{2} \nabla \times \nabla \times\left(\dot{W} \nabla \dot{W}^{*}\right)$, so that it is possible to write:

$$
\begin{aligned}
\vec{P}(x, y)= & \frac{D}{2 \omega} \Im\left\{\nabla\left(\nabla^{2} \dot{W}\right) \dot{W}^{*}-\nabla^{2} \dot{W} \nabla \dot{W}^{*}\right. \\
& \left.-\frac{(1-v)}{2} \nabla \times \nabla \times\left(\dot{W} \nabla \dot{W}^{*}\right)\right\} .
\end{aligned}
$$

Using the hypotheses of propagating, non-interactive waves, the third term in Eq. (21) vanishes, and the expression of the power flow is elegantly simplified to:

$$
\vec{P}(x, y)=\sqrt{D \rho h} \Im\left\{\dot{W} \nabla \dot{W}^{*}\right\}
$$

which, in terms of accelerations, can be written as:

$$
\vec{P}(x, y)=\frac{D\left(k_{x}^{2}+k_{y}^{2}\right)}{\omega^{3}} \Im\left\{\ddot{W} \nabla \ddot{W}^{*}\right\}
$$

It is important to point out at this point that this result does not agree exactly with Eqs (19). The difference is a scalar coefficient which equals $\left(k_{x}^{2}+v k_{y}^{2}\right) /\left(k_{x}^{2}+k_{y}^{2}\right)$ in the $x$ direction and $\left(k_{y}^{2}+v k_{x}^{2}\right) /\left(k_{x}^{2}+k_{y}^{2}\right)$ in the $y$ direction.

Carniel [4] proposes a slightly different formulation, which leads to a different finite-difference approximation. Starting from Eq. (21), assuming a free-field propagation, and using Eqs (4), it is possible to write:

$$
\vec{P}(x, y)=-\frac{D}{\omega^{3}} \Im\left\{\nabla^{2} \ddot{W} \nabla \ddot{W}^{*}\right\}
$$

\section{Finite difference approximations}

Now we will introduce the finite difference approximations in the derived formulae. This will yield the mathematical expressions which can be used to compute the power flow in plates using measured data.

Not only accelerometers can be used to measure structural power flow. Strain gages [7], laser holography [9], and laser vibrometry [3] have also been used. In this study we will assume that only out-of-plane accelerations or velocities at neighboring points can be measured on a structure surface. This assumption is appropriate whenever accelerometers or laser vibrometers are used. The frequency domain formulation used here makes it straightforward to transform velocities into accelerations and vice-versa.

In this section we will use a basic measurement cell, consisting of nine acceleration measurements arranged 


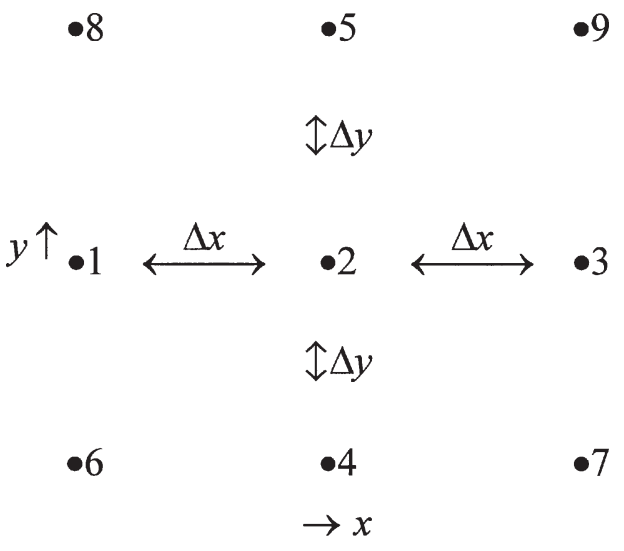

Fig. 1. Measurement cell configuration.

as a $3 \times 3$ matrix with spacing $\Delta x$ in the $x$ direction and $\Delta y$ in the $y$ direction. Figure 1 shows the measurement cell and indicates the measurement point numbering adopted.

The cross spectrum of two measured accelerations, $\ddot{W}_{i}$ and $\ddot{W}_{j}$, will be denoted by:

$$
E\left[\ddot{W}_{i}^{*} \ddot{W}_{j}\right]=S_{i j}
$$

Note that this is not rigorously the cross-power spectral density. To know exactly how $S_{i j}(\omega)$ should be interpreted, one needs to have in mind that $\ddot{W}(\omega)$ is the complex amplitude of a sinusoidal component, which is equal to twice the corresponding complex Discrete Fourier Transform (DFT) coefficient. Therefore, when one is dealing with random signals, $S(\omega)$ of Eq. (25) is four times the spectral density multiplied by the frequency resolution $\Delta f$, where $f$ is the frequency in Hertz. The finite difference approximations of the spatial derivatives used here are:

$$
\begin{aligned}
\frac{\partial \ddot{W}}{\partial x} & =\frac{\ddot{W}_{3}-\ddot{W}_{1}}{2 \Delta x} \text { (central) or } \\
& =\frac{\ddot{W}_{3}-\ddot{W}_{2}}{\Delta x} \text { (right), } \\
\frac{\partial \ddot{W}}{\partial y} & =\frac{\ddot{W}_{5}-\ddot{W}_{4}}{2 \Delta y} \text { (central) or } \\
& =\frac{\ddot{W}_{5}-\ddot{W}_{2}}{\Delta y}(\text { right }), \\
\nabla^{2} \ddot{W} & =\left(\Delta x^{2}\left(W_{5}+W_{4}-2 W_{2}\right)\right. \\
& \left.+\Delta y^{2}\left(W_{3}+W_{1}-2 W_{2}\right)\right) / \Delta x^{2} \Delta y^{2}, \\
\frac{\partial^{2} \ddot{W}}{\partial x \partial y} & =\frac{\left(\ddot{W}_{6}+\ddot{W}_{9}\right)-\left(\ddot{W}_{7}+\ddot{W}_{8}\right)}{4 \Delta x \Delta y} .
\end{aligned}
$$

Applying the above relations to the formulae derived in the previous section, the different methods for computing structural power flow in plates can be obtained.

Three-accelerometer, two cross-spectra method: Starting from Eq. (23) and using the right side finite differences in the first two equations in Eqs (26), it is possible to write:

$$
\begin{aligned}
& P_{x} \cong \frac{\sqrt{D \rho h}}{\omega^{2} \Delta x} \Im\left\{-S_{23}\right\}, \\
& P_{y} \cong \frac{\sqrt{D \rho h}}{\omega^{2} \Delta y} \Im\left\{-S_{25}\right\} .
\end{aligned}
$$

Five-accelerometer, four cross-spectra method: Starting from Eq. (23) and using the central finite differences in the first two equations in Eqs (26), it is possible to write:

$$
\begin{aligned}
& P_{x} \cong \frac{\sqrt{D \rho h}}{2 \omega^{2} \Delta x} \Im\left\{S_{21}-S_{23}\right\}, \\
& P_{y} \cong \frac{\sqrt{D \rho h}}{2 \omega^{2} \Delta y} \Im\left\{S_{24}-S_{25}\right\} .
\end{aligned}
$$

Five-accelerometer, ten cross-spectra method: Starting from Eq. (24) and using the finite-difference approximations in Eqs (26), it is possible to write:

$$
\begin{aligned}
& P_{x} \cong \frac{D}{2 \omega^{2} \Delta x^{3} \Delta y^{2}} \Im\left\{2 \Delta y^{2}\left(S_{21}-S_{23}+S_{13}\right)\right. \\
& \left.+\Delta x^{2}\left[2\left(S_{21}-S_{23}\right)+S_{53}+S_{43}+S_{15}+S_{14}\right]\right\}, \\
& P_{y} \simeq \frac{D}{2 \omega^{2} \Delta y^{3} \Delta x^{2}} \Im\left\{2 \Delta x^{2}\left(S_{24}-S_{25}+S_{45}\right)\right. \\
& \left.+\Delta y^{2}\left[2\left(S_{24}-S_{25}\right)+S_{35}+S_{43}+S_{15}+S_{41}\right]\right\} .
\end{aligned}
$$

Nine-accelerometer, twenty cross-spectra method: Starting from Eqs (11)-(13), and using the finite difference approximations in Eqs (26), it is possible to write:

$$
\begin{aligned}
& P_{x}^{b}(x, y)=\frac{D\left(k_{x}^{2}+v k_{y}^{2}\right)}{4 \omega^{3} \Delta x} \Im\left\{S_{21}-S_{23}\right\}, \\
& P_{y}^{b}(x, y)=\frac{D\left(k_{y}^{2}+v k_{x}^{2}\right)}{4 \omega^{3} \Delta y} \Im\left\{S_{24}-S_{25}\right\}, \\
& P_{x}^{S}(x, y)=\frac{D\left(k_{x}^{2}+k_{y}^{2}\right)}{4 \omega^{3} \Delta x} \Im\left\{S_{21}-S_{23}\right\}, \\
& P_{y}^{S}(x, y)=\frac{D\left(k_{x}^{2}+k_{y}^{2}\right)}{4 \omega^{3} \Delta y} \Im\left\{S_{24}-S_{25}\right\},
\end{aligned}
$$




$$
\begin{aligned}
P_{x}^{t}(x, y)= & -\frac{D(1-v)}{16 \omega^{3} \Delta x \Delta y^{2}} \Im\left\{S_{56}+S_{59}-S_{57}\right. \\
& \left.-S_{58}-S_{46}-S_{49}+S_{47}+S_{48}\right\}, \\
P_{y}^{t}(x, y)= & -\frac{D(1-v)}{16 \omega^{3} \Delta y \Delta x^{2}} \Im\left\{S_{36}+S_{39}-S_{37}\right. \\
& \left.-S_{38}-S_{16}-S_{19}+S_{17}+S_{18}\right\} .
\end{aligned}
$$

The dispersion relation can also be computed with the finite-difference approximations above. Starting from Eqs (4), one can write:

$$
\frac{\partial^{2} \ddot{W}}{\partial x^{2}}=-k_{x}^{2} \ddot{W} \cong \frac{\ddot{W}_{3}+\ddot{W}_{1}-2 \ddot{W}_{2}}{\Delta x^{2}} .
$$

Pre and post multiplying this expression by $\ddot{W}_{2}^{*}$ and using Eq (25), it is straightforward to write:

$$
S_{21}+S_{23}-2 S_{22} \cong-k_{x}^{2} \Delta x^{2} S_{22}
$$

or, finally,

$$
\begin{aligned}
& k_{x}=\frac{1}{\Delta x} \sqrt{\frac{2 S_{22}-S_{21}-S_{23}}{S_{22}}}, \\
& k_{y}=\frac{1}{\Delta y} \sqrt{\frac{2 S_{22}-S_{24}-S_{25}}{S_{22}}},
\end{aligned}
$$

where the expression for $k_{y}$ was obtained analogously. The expression for $k$ can be obtained with $k^{2}=k_{x}^{2}+k_{y}^{2}$ or using the finite-difference approximation for the Laplacian. This expression is identical to the expression derived by Carniel [4] for beams. He has shown that this estimation of the wavenumber may be corrected to account for the finite length $\Delta x$ with:

$$
\begin{aligned}
& k_{x}=\frac{2}{\Delta x} \sin ^{-1}\left(\frac{k_{x} \Delta x}{2}\right), \\
& k_{y}=\frac{2}{\Delta y} \sin ^{-1}\left(\frac{k_{y} \Delta y}{2}\right) .
\end{aligned}
$$

The computed power flow may be compared with the input power. If there is no energy dissipation within the plate (no material damping), the power flow integrated in a closed contour encompassing the excitation point (without any dissipation device) must be equal to the input power. The power input by an external, con- centrated harmonic force $F(t)$ is given by:

$$
\begin{aligned}
\left\langle P_{i n}\right\rangle_{t} & =\frac{1}{2} \Re\left\{F(\omega) \dot{W}(\omega)^{*}\right\} \\
& =\frac{1}{2} \Re\left\{F(\omega)\left(\frac{\ddot{W}(\omega)}{\mathrm{i} \omega}\right)^{*}\right\} \\
& =\frac{1}{2 \omega} \Im\left\{S_{F \ddot{W}}(\omega)\right\},
\end{aligned}
$$

where $S_{F \ddot{W}}(\omega)$ is the cross spectrum between the excitation force and the acceleration at the excitation point location.

\section{Regressive two-dimensional Fourier series}

Two-dimensional velocity fields measured over the surface of a structure at a given frequency, given by amplitudes and phases measured relative to a reference signal (which can be the velocity at a given location) are usually referred to as Operational Deflection Shapes (ODS) or, when the reference signal is the input force, mobility shapes. ODS's are frequently measured over rectangular grids using scanning laser Doppler vibrometers. If a two-dimensional Fourier series model is used to interpolate or approximate the measured data, the partial spatial derivatives required to compute the structural power flow may be easily computed in the wavenumber domain.

Two-dimensional ODS's measured over equallyspaced rectangular grids, say $H_{m n}(\omega)$, can be interpolated using the two-dimensional Discrete Fourier Transform (DFT). The difficulty with using the DFT is that its implicit periodization introduces high-frequency components that account for the sharp edges present in the wrapped-around data. This phenomenon is known as leakage. In the data smoothing process, leakage is prejudicial, as it causes distortion of the lowpass filtered data. When dealing with plate vibrations, this problem does not exist for clamped boundaries, is not too serious in the case of simply supported boundaries, but becomes critical in the case of free boundaries. It should be noted that the boundaries can be actual boundary conditions or just measurement field boundaries.

The usual way to reduce leakage is windowing, but this technique is not suitable in the case of finite length, spatial domain data. To overcome the leakage problem, the proposed technique consists of representing the data by a two-dimensional regressive discrete Fourier 
series (RDFS) proposed by Arruda [1], which will be briefly reviewed here. Unlike the DFT, in the RDFS the original length of the data is not assumed to be equal to the signal period nor is the number of frequency lines assumed to be equal to the number of data points. With the two-dimensional, equally-spaced RDFS model, the mobility shape $H_{m n}$ (where the frequency dependency is omitted for simplicity) is expressed as:

$$
\begin{aligned}
H_{m n} & =\sum_{k=-p}^{p} \sum_{l=-q}^{q} Z_{k l} W_{\mathrm{M}}^{m k} W_{\mathrm{N}}^{\mathrm{ln}}+\varepsilon_{m n} ; \\
m & =0, \ldots, M-1 ; n=0, \ldots, N-1 ;
\end{aligned}
$$

where $H_{m n}$ represents the discretized data with constant spatial resolutions $\Delta x$ and $\Delta y, W_{\mathrm{M}}=\exp (\mathrm{i} 2 \pi / \mathrm{M})$, $W_{\mathrm{N}}=\exp (\mathrm{i} 2 \pi / \mathrm{N})$, and $\varepsilon_{m n}$ accounts for the noise and higher frequency contents of $H$. Note that $M \neq \mathrm{M}$ and $N \neq \mathrm{N}$. The length of the data in $x$ is $M \Delta x$, but the period of the RDFS is $\mathrm{M} \Delta x>M \Delta x$. Data reduction is achieved because $p \ll M$ due to the expected low wave number of the mobility shape surface. In the $y$ direction $\mathrm{N} \Delta y>N \Delta y$ and $q \ll N$. The $M \times N$ data in $\mathbf{H}$ are represented by a $(2 p+1) \times(2 q+1)$ complex matrix $\mathbf{Z}$ of elements $Z_{k l}$.

The RDFS is an approximation instead of an interpolation of $H_{m n}$. Thus, the Euler-Fourier coefficients cannot be calculated by the DFT. Rewriting Eq. (35) in matrix form:

$$
\mathbf{H}=\mathbf{W}_{\mathbf{M}} \mathbf{Z} \mathbf{W}_{\mathrm{N}}+\varepsilon .
$$

The least-squares solution is given by:

$$
\mathbf{Z}=\left(\mathbf{W}_{\mathbf{M}}^{H} \mathbf{W}_{\mathbf{M}}\right)^{-1} \mathbf{W}_{\mathbf{M}}^{H} \mathbf{H} \mathbf{W}_{\mathrm{N}}^{H}\left(\mathbf{W}_{\mathrm{N}} \mathbf{W}_{\mathrm{N}}^{H}\right)^{-1},
$$

where the matrices to be inverted have a very small size, $(2 p+1) \times(2 p+1)$ and $(2 q+1) \times(2 q+1)$, respectively, and ${ }^{H}$ denotes the matrix complex conjugate. The smoothed data $\mathbf{H}^{(s)}$ may be obtained from:

$$
\mathbf{H}^{(s)}=\mathbf{W}_{\mathrm{M}} \mathbf{Z} \mathbf{W}_{\mathrm{N}},
$$

where $W_{\mathrm{M}}$ and $W_{\mathrm{N}}$ can be calculated for the desired spatial resolution. The reduction of the data is achieved as $\mathbf{Z}$ represents the data using only $(2 p+1)(2 q+1)$ values, instead of the original MN values. The formulation of the RDFS for non-equally spaced data [1] can be used in the place of the formulation above when the mobility shapes are mapped over non-regular, arbitrary grids, while the DFT is only applicable to equally-

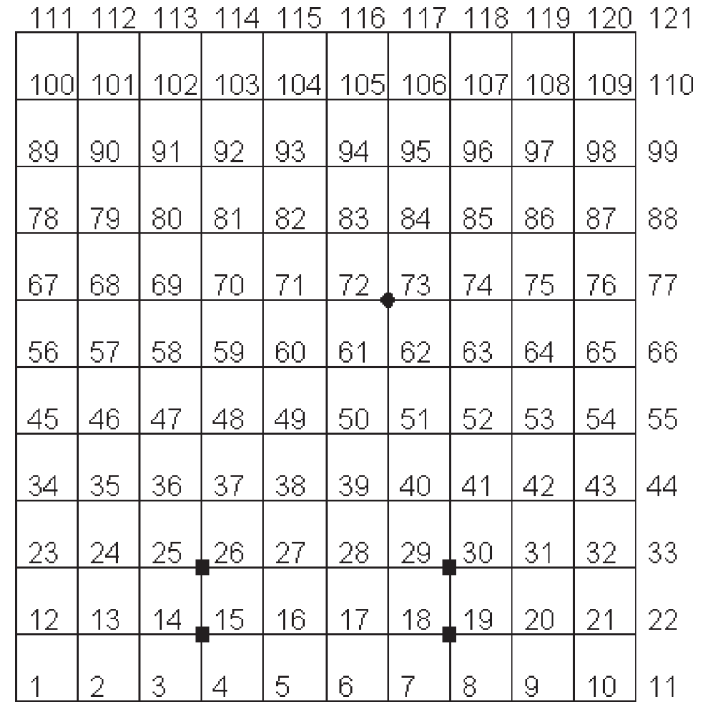

Fig. 2. FE mesh of the rectangular plate indicating the location of the rubber mounts (squares) and exciter (diamond).

spaced, rectangular grids. The spatial derivatives are obtained simply by multiplying each coefficient $Z_{k l}$ by the corresponding wavenumber, multiplied by the imaginary unit to the appropriate power.

\section{Numerical results}

In order to compare the different methods for computing the structural power flow in thin plates exposed in the previous sections, the responses of a rectangular plate with localized, "sky-hooked" springs and dampers excited by point forces were simulated using a Finite Element model. Rectangular, non-conforming thin plate elements with polynomial interpolation functions of incomplete quartic order were used [12].

The simulated plate is a $0.45 \mathrm{~m} \times 0.40 \mathrm{~m}, 1 \mathrm{~mm}$ thick aluminum plate. The plate was modeled with one hundred finite elements, ten in each orthogonal direction. Different configurations with dampers attached at different locations and excitation at different points were simulated, but only one typical case, corresponding to the experimental set-up shown in the next section, is shown here.

Four rubber mounts were simulated using "skyhooked" springs and dash-pots attached at FE nodes. The nodes where the rubber mounts are located are indicated in Fig. 2. The values of the stiffness and damping coefficients used in the simulation were obtained from real cylindrical rubber mounts $(1 \mathrm{~cm}$ diameter, $1 \mathrm{~cm}$ long, with M4 bolts on each side) which 
were later used in the experimental setup. The relevant stiffness and damping coefficients of the rubber mounts were identified by mounting a block of aluminum with known inertial characteristics on top of it and measuring the resulting natural frequencies. The identified longitudinal stiffness value was $1.0 \times 10^{5} \mathrm{~N} \mathrm{~m}^{-1}$ and the corresponding damping value $23 \mathrm{~N} \mathrm{~s} \mathrm{~m}^{-1}$. The estimated bending angular stiffness value was $4.5 \mathrm{~N} \mathrm{~m} \mathrm{rad}^{-1}$ and the corresponding damping value $2.0 \times 10^{-3} \mathrm{~N} \mathrm{~m} \mathrm{~s} \mathrm{rad}^{-1}$. A unity force excitation, placed at the location indicated in Fig. 2, of the plate was used as the energy source. The plate was modeled without internal damping, such that all the energy dissipation occurs at the rubber mounts, which is a reasonably realistic assumption for an aluminum plate mounted on rubber isolators. Lumped masses were added to account for the mass loading caused by the rubber mounts and the shaker attachment on the plate. The added mass values were roughly estimated from the geometry and weight of the parts. The lumpedmass values used in the FE model were $5 \times 10^{-3} \mathrm{~kg}$ translational mass and $5 \times 10^{-9} \mathrm{~kg} \mathrm{~m}^{2}$ rotational inertia for the rubber mounts and $3 \times 10^{-3} \mathrm{~kg}$ translational mass and $2 \times 10^{-6} \mathrm{~kg} \mathrm{~m}^{2}$ rotational inertia for the shaker attachment (disk bonded to the structure, piezoelectric force transducer, and stinger holder).

Initially the plate responses were computed in the frequency range from 50 to $200 \mathrm{~Hz}$ with a $2 \mathrm{~Hz}$ frequency resolution. These responses can be interpreted as responses to a harmonic excitation at each frequency, as responses to a constant amplitude multisine excitation, or as responses to a white noise random excitation, in which case the responses should be seen as cross spectral densities between the response degrees of freedom and the excitation force with unitary autospectrum.

Guyan reduction was used to save computational time when solving the eigenvalue problem. The reduced mass and stiffness matrices were assembled into a generalized, non-proportionally damped doubleorder model where the damping matrix was formed by inserting damping diagonal elements. The stiffness of the rubber mounts was modeled by adding their spring constants to the corresponding stiffness matrix diagonal elements. The lumped masses due to the massloading effect of the rubber mounts and shaker attachment were also included by adding theirs values to the appropriate diagonal elements of the mass matrix. After solving the eigenproblem, the eigenvalues were expanded to generate 3 degrees of freedom per node (one transverse displacement and two orthogonal rotations),

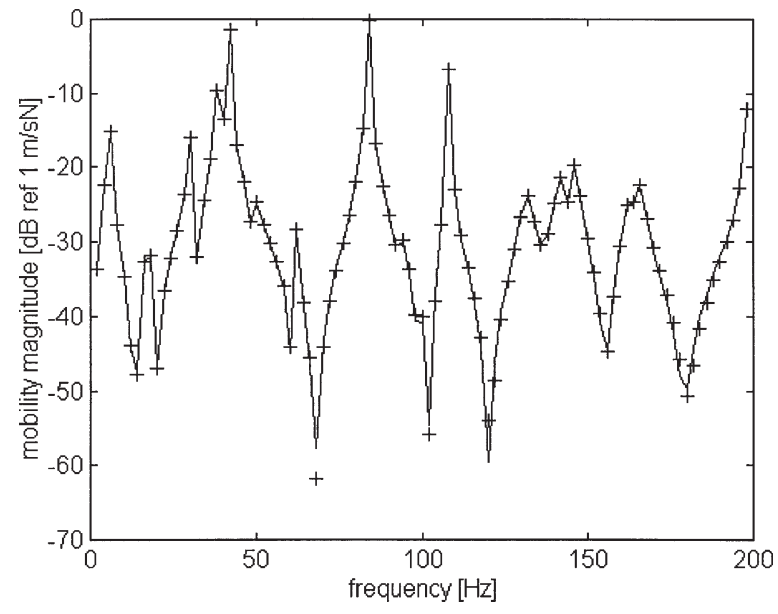

Fig. 3. Driving-point mobility. - with and ++ without residual flexibility compensation.

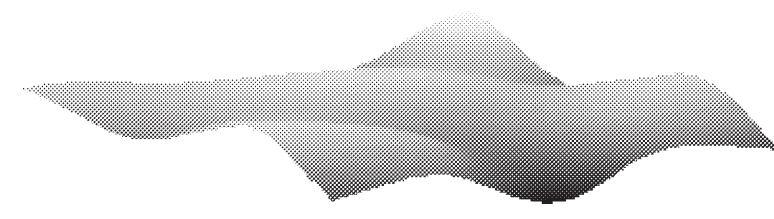

(a)

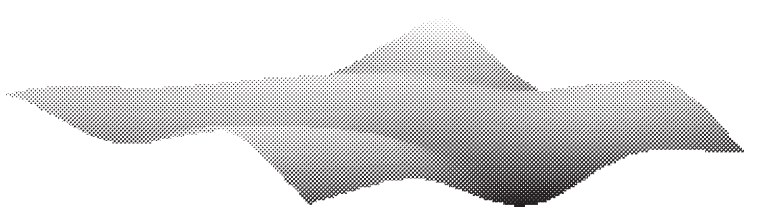

(b)

Fig. 4. Mobility ODS at $128 \mathrm{~Hz}$. (a) FE prediction; (b) approximated using a 2-D RDFS with $p=q=4, \mathrm{M} / M=\mathrm{N} / N=1.5$.

which were necessary to compute responses at arbitrary points using the FE interpolation functions.

Figure 3 shows the driving-point mobility in the frequency range investigated. The FRF's were computed by modal superposition using the first 60 modes. The influence of the residual flexibility of the higher order modes in the frequency range of interest is very small, as it can be seen in Fig. 3, where the FRF's obtained with and without residual flexibility compensation are plotted. Although this difference is small, it is important to include the residual flexibility compensation when computing the power flow, as it was shown by Gavric and Pavic [6].

The power flow was computed from the simulated responses using the finite-difference methods exposed previously, using the RDFS-based method proposed in 


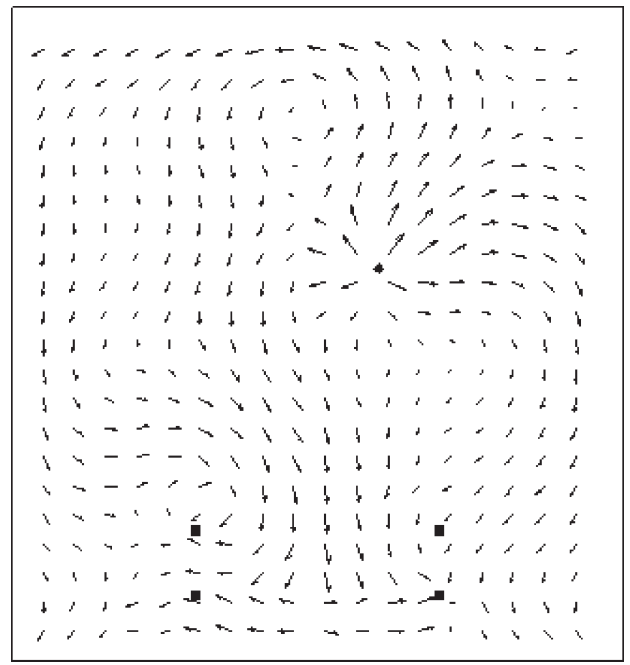

(a)

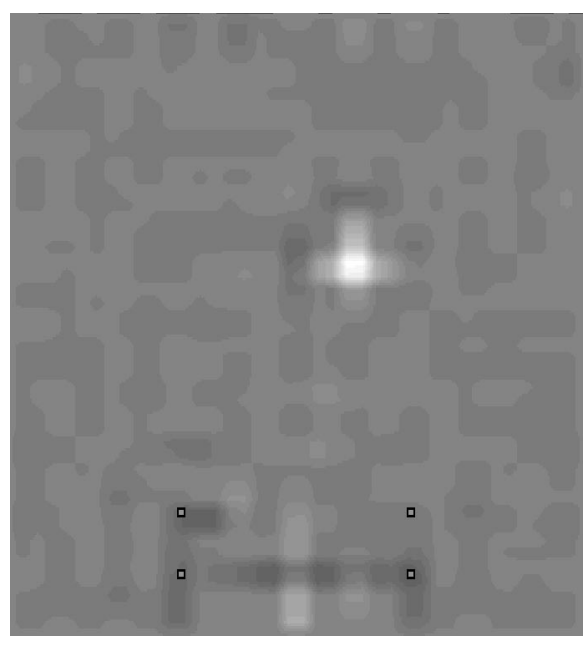

(c)

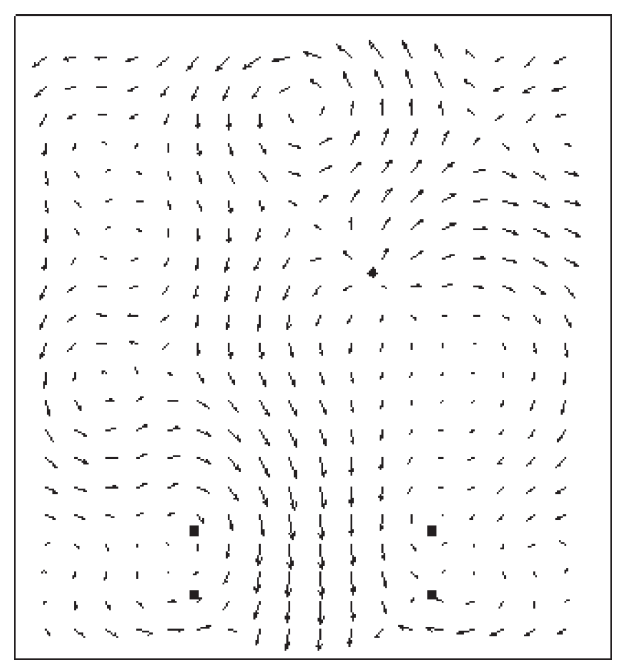

(b)

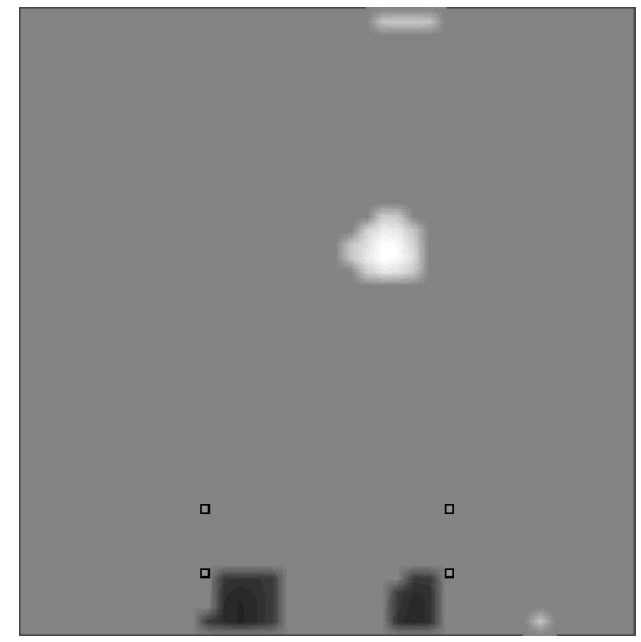

(d)

Fig. 5. Structural power flow arrow maps and divergence plots computed from simulated ODS using FEM interpolation functions. (a) and (c) Eq. (21); (b) and (d) Eq. (22).

this paper, and using the FE interpolation functions directly. Typical results are shown and discussed in what follows. The mobility ODS predicted using the FE model was interpolated using the FE interpolation functions to increase the spatial resolution from the original $11 \times 11$ to $46 \times 40$ (the larger resolution along the longer side of the plate, along the $y$ direction).

Figure 4 shows the mobility ODS simulated with the FE model at $128 \mathrm{~Hz}$. Figure 5 and Fig. 6 show the power flow computed at this frequency using the methods previously exposed. The arrows indicate the direction of the structural power flow and its relative mag- nitude. To enhance the visualization of the power flow pattern, square root magnitudes were used. With a linear magnitude scale the smaller arrows cannot be visualized and it becomes more difficult to understand the flow pattern. To take the square root of the magnitude without changing the flow direction, the following equations were used:

$$
P=P_{x}+\mathrm{i} P_{y}, \quad \bar{P}=\sqrt{|P|} \mathrm{e}^{\mathrm{i} \operatorname{atan}\left(P_{y} / P_{x}\right)} .
$$

The magnitudes of the computed power flow are verified later in plots of magnitude against frequency, 


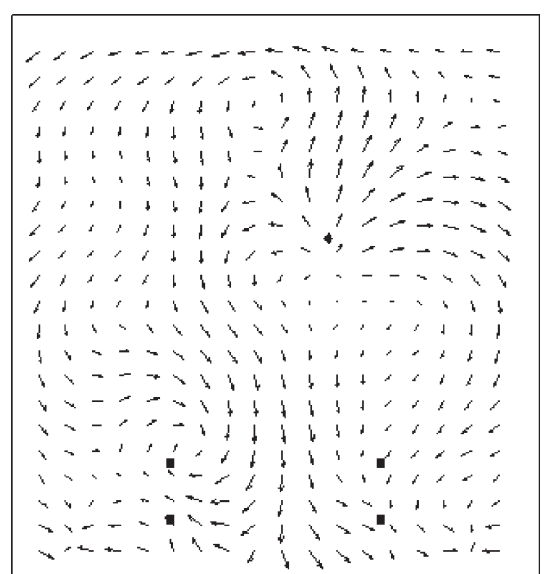

(a)

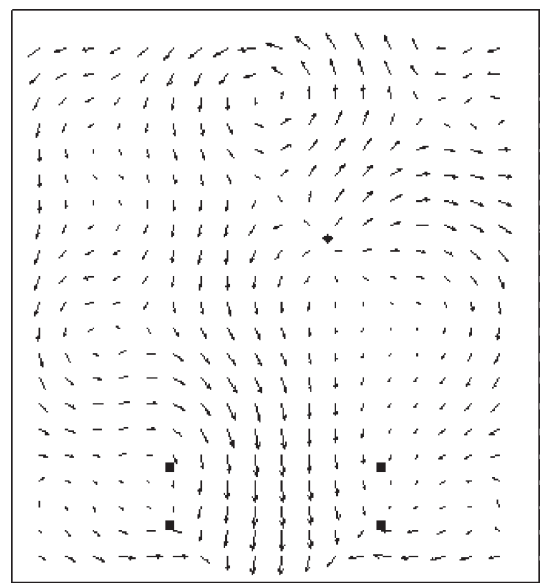

(c)

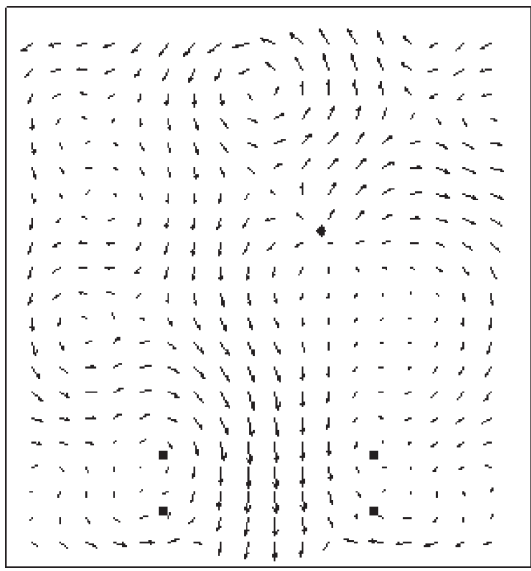

(e)

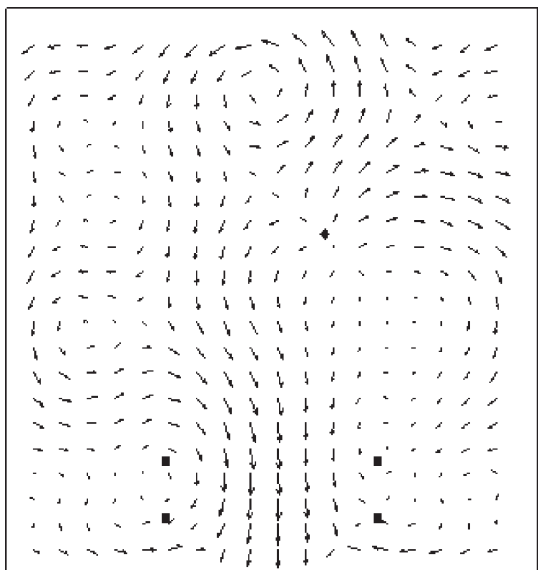

(b)

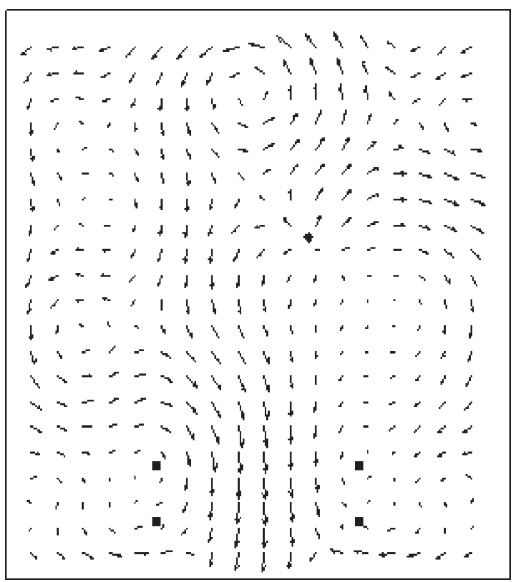

(d)

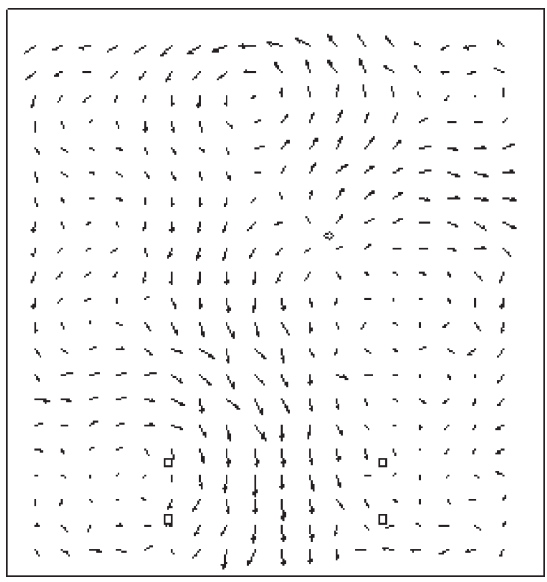

(f)

Fig. 6. Structural power flow at $128 \mathrm{~Hz}$ computed using different methods (a) RDFS and Eq. (21); (b) RDFS and Eq. (22); (c) finite differences Eq. (27); (d) Eq. (28); (e) Eq. (29); (f) Eqs (30a)-(30c). 


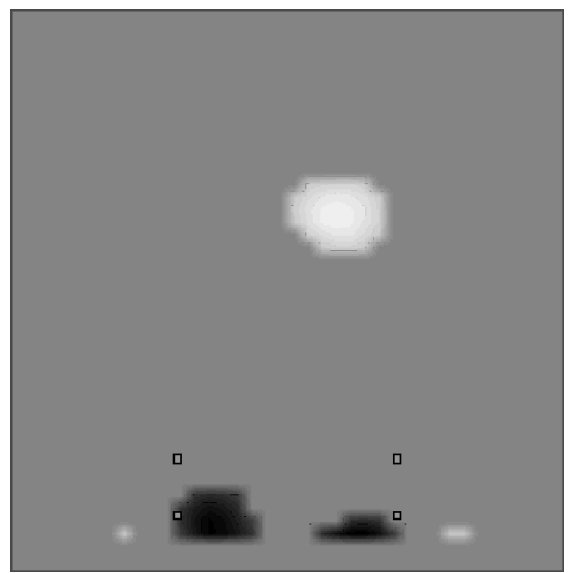

(a)

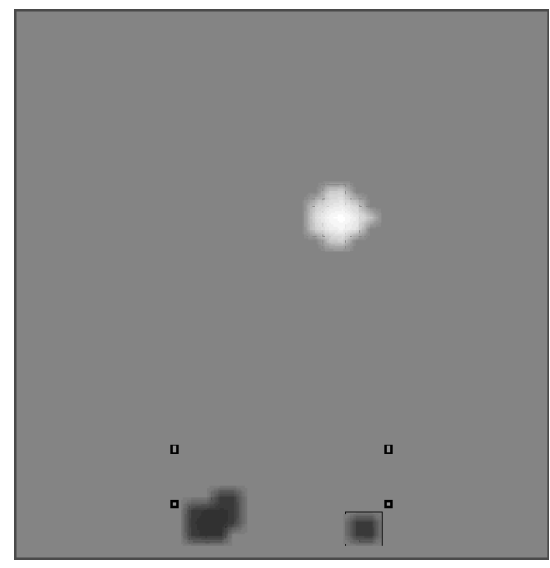

(c)

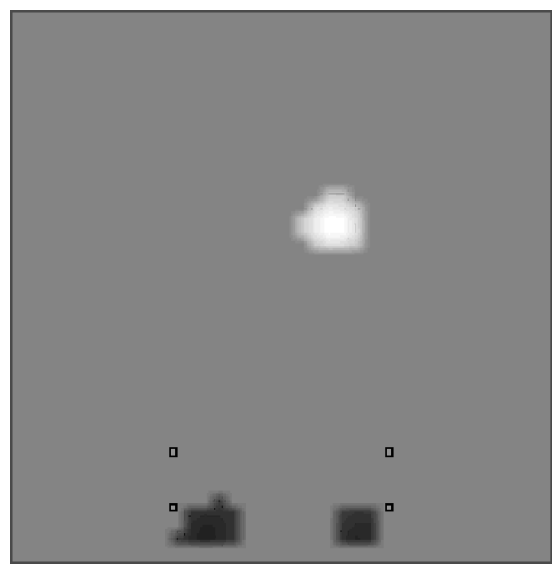

(e)

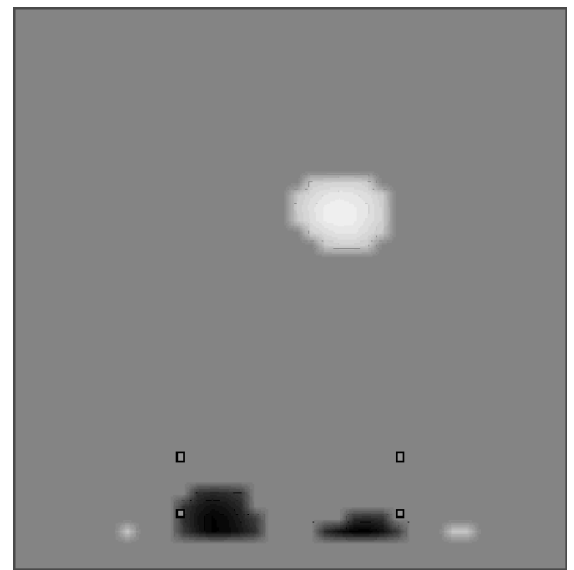

(b)

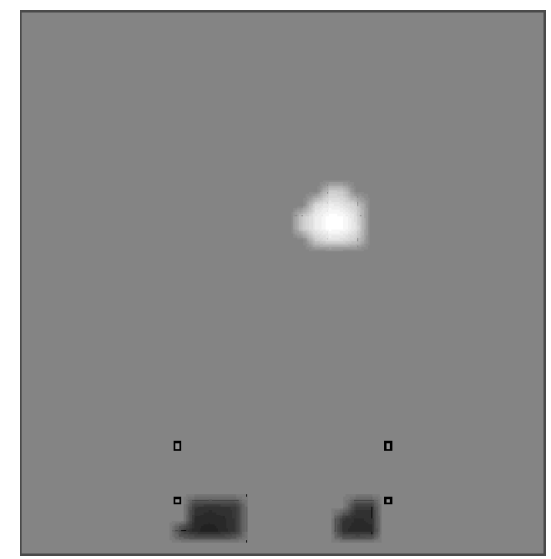

(d)

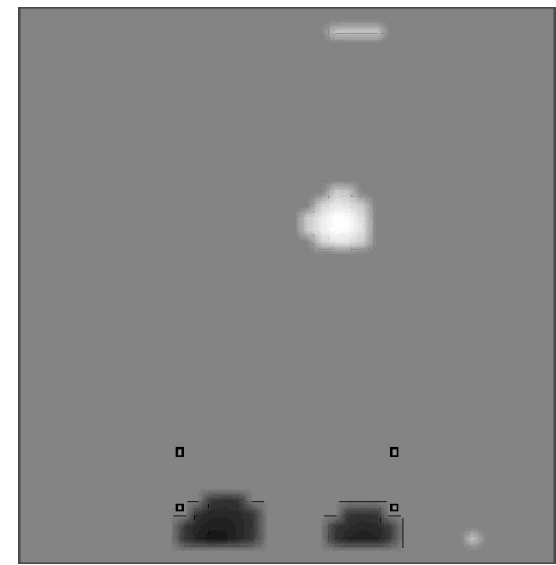

(f)

Fig. 7. Divergence plots obtained from the power flow computed with: RDFS approximation using (a) Eq. (21) and (b) Eq. (22); finite differences using (c) Eq. (27); (d) Eq. (28); (e) Eq. (29); (f) Eqs (30a)-(30c). 


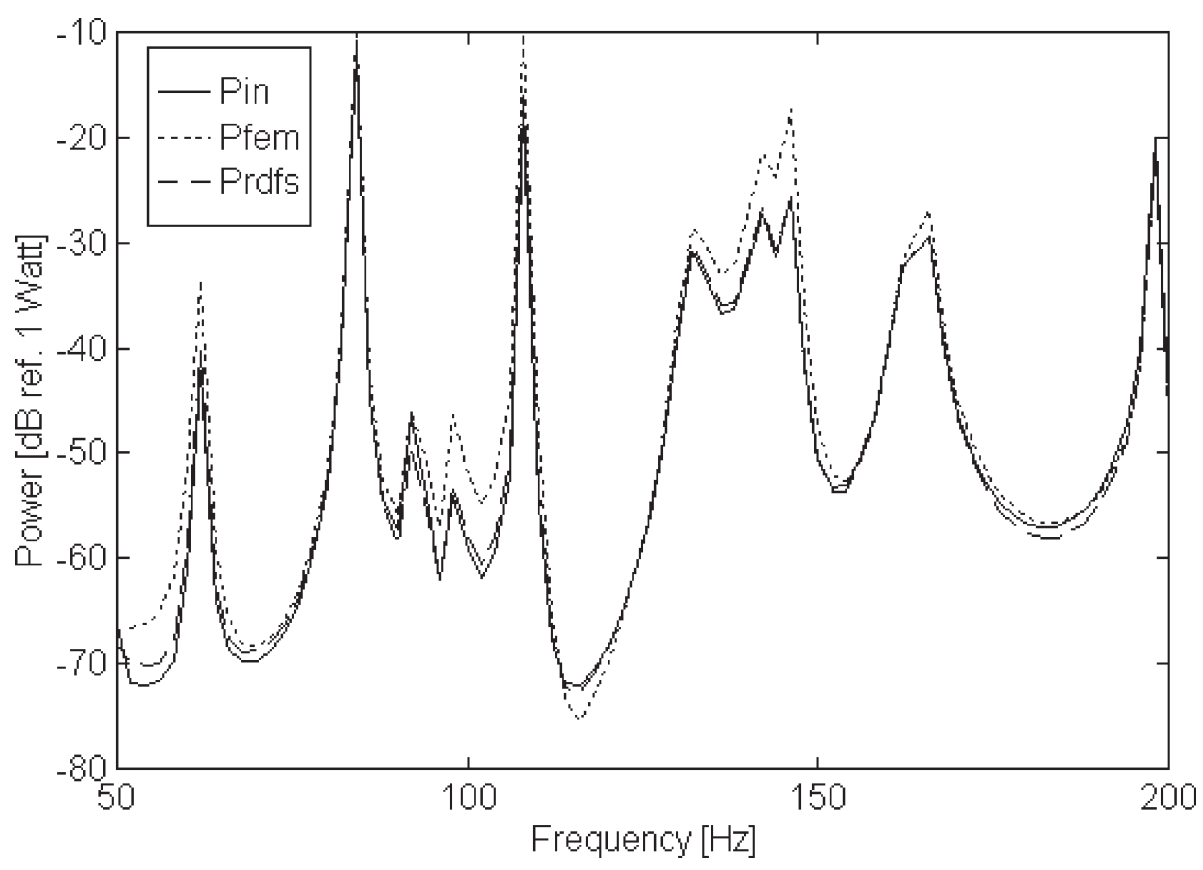

(a)

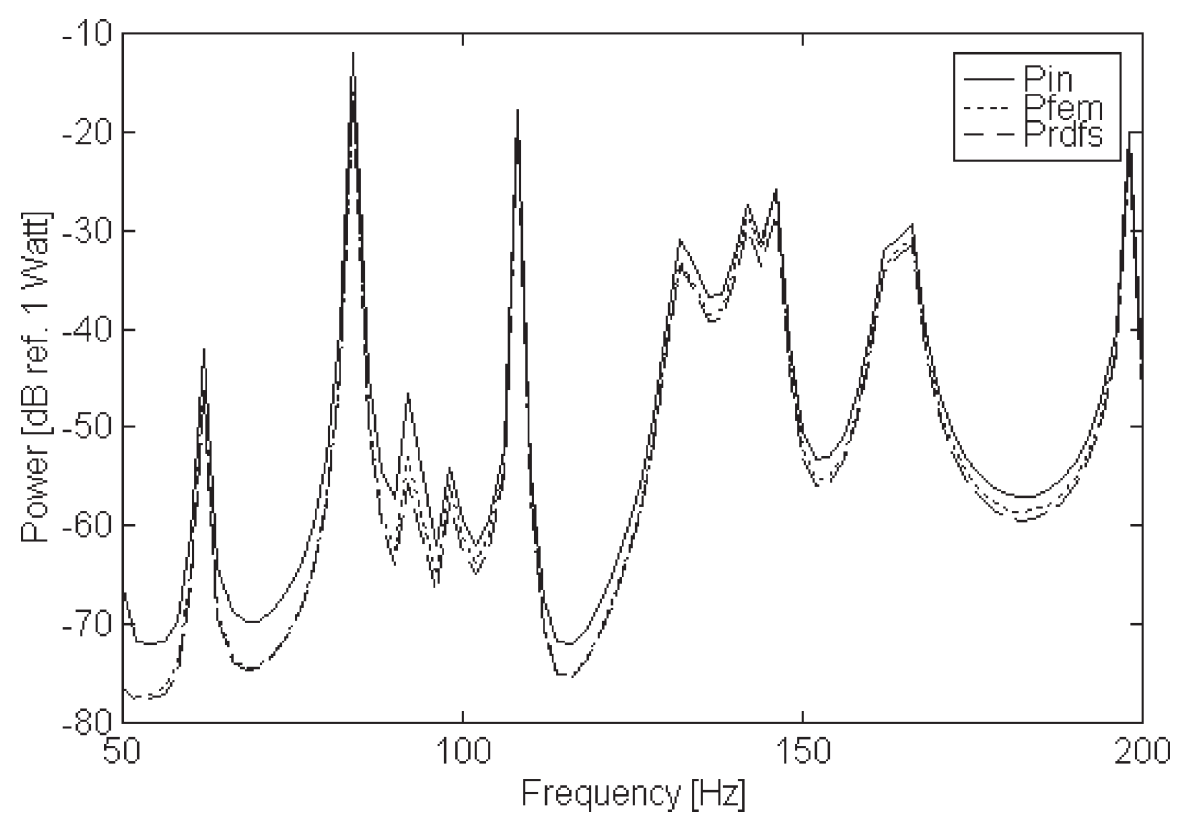

(b)

Fig. 8. Input power versus power flow across a closed path encircling the shaker location. (a) Power flow computed using Eq. (21); (b) using Eq. (22).

comparing the input power with the power flow integrated over a path encircling the excitation location.

Figure 5 shows the power flow arrow maps and the divergence plots obtained from the simulated ODS us- ing the FE interpolation functions directly. As the second order derivatives of the interpolation function of the finite element used are not continuous across element borders, the higher order terms in Eq. (10) com- 
puted to take into account the near-field effects may have significant errors. Therefore, the results computed with the FE interpolation functions cannot be taken as exact when comparing with results obtained with the other methods. The power flow was computed with and without the far field assumption using the FE interpolation functions, Eqs (22) and (21), respectively.

In Fig. 6 it can be seen that the pattern of the power flow is equivalent for all the methods used. A coarser grid of $22 \times 20$ was used to improve the visualization of the power flow arrow maps.

In this example, the location of the energy source and the energy sinks are clearly indicated by the power flow pattern. However, at higher frequencies, where the power flow patterns can be much more complicated, it may become difficult to localize sources and sinks. Another way to visualize sources and sinks is to compute the power flow across a closed path around each location on the structure surface. The resulting value is the net power variation at the location, which is positive if there is an energy source at the location, negative if there is a sink, and zero if there is no source or sink. This is equivalent to computing the divergence of the vibration intensity field, which was proposed by Pascal et al. [10], and, therefore, equivalent to computing a spatial derivative of the computed intensity field. A further derivative could amplify noise, but the averaging effect of integrating along a closed path around each location alleviates this problem. However, one must have this in mind when interpreting the results. Figures 5 and 7 show divergence plots computed at $128 \mathrm{~Hz}$ using the previously computed intensity fields (power flow distributions).

It is clear from Figs 5(c)-(d) and Figs 7(a)-(b) that the divergence plots computed from power flow fields obtained using the far-field assumption indicate more clearly the locations of the source and the sinks. This is probably due to the problems involved in computing the higher-order derivatives of the near-field contributions to the power flow, both using the FE nonconforming interpolation functions and the RDFS approximation. The localization of the sinks is more difficult in this example, as the energy dissipation is spread through four different locations, while the energy input is all concentrated at one location. All the finitedifference based methods produced divergence plots (Figs 7(c)-(f)) that allow the localization of the source and sinks.

In the RDFS approximation, as the area between the rubber mounts has a higher wavenumber content, to approximate it more exactly, more RDFS lines would be needed. However, increasing the number of lines in the RDFS tends to increase the errors at the sides of the plate due to the minimized, but still present, leakage effect. When using experimental data the effect of using a higher number of frequency lines is even worse, as it increases the effect of noise in the spatial derivatives. The influence of this leakage is more detrimental to the power flow computed using Eq. (22), for it involves higher order derivatives.

Finally, to verify the magnitudes of the computed power flow fields, the power integrated along a path encircling the shaker location is compared with the input power computed from the driving-point impedance. Figure 8 shows the input power and the power flow computed over the whole frequency range investigated. Results in Fig. 8 show that the inclusion of the nearfield effects improve the power flow amplitude precision near the shaker location (the path used consisted of a rectangle of dimensions $8 \times 8 \mathrm{~cm}$ ). As expected, the results obtained using the power flow computed under the far-field assumption get poorer as the frequency decreases. It also shows that the power flow computed using the RDFS approximation is closer to the exact input power value than the power flow computed using the FE interpolation functions.

\section{Experimental results}

A system with the characteristics of the system used in the numerical simulation was build and tested using a scanning laser Doppler vibrometer (LDV). The shaker was attached to the back of the plate using a small steel disk glued (Hotinger X-60 glue) to the structure. A piezoelectric force transducer $(2.27 \mathrm{mV} / \mathrm{N}$, PCB model 208A03) was screwed to the disk and connected to the shaker using a stinger holder and a suitable stinger ( $1.5 \mathrm{~mm}$ diameter, $90 \mathrm{~mm}$ long made of a copper alloy). The shaker used was a small modalanalysis type shaker (Bruel \& Kjaer model 4810). The shaker was supported by a frame structure standing on tripods. The rubber mounts were attached to a thick aluminum plate $(355 \times 129 \times 8 \mathrm{~mm})$ which was bolted to a large steel block, measuring approximately $0.32 \times 0.4 \times 0.24 \mathrm{~m}$. The characteristics of the plate and rubber mounts have already been described in the previous section. The scanning laser vibrometer was standing on a tripod at approximately $2 \mathrm{~m}$ from the structure, aiming at its center. The sensitivity of the laser vibrometer (Ometron model VS100) was set to $100 \mathrm{mV} / \mathrm{mm} / \mathrm{s}$ and the low pass filter cutoff frequency 


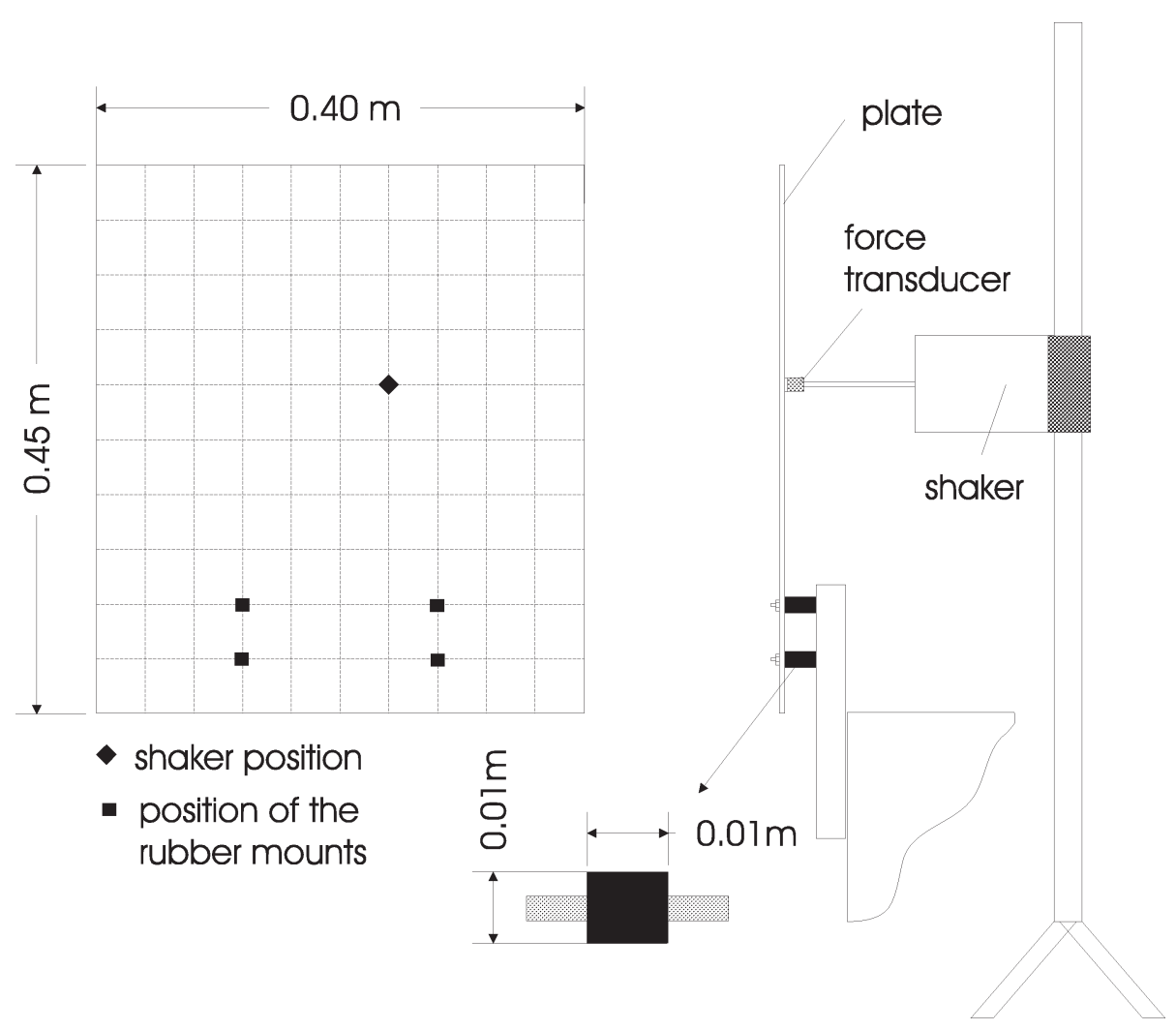

Fig. 9. Experimental setup.

was set to $5 \mathrm{kHz}$. Figure 9 shows a scheme of the experimental setup. The scanning hardware and software, developed at the PMA Division of K.U. Leuven, was programmed so as to scan a rectangular area with an equally-spaced measurement grid of $46 \times 40$. It was not necessary to compensate for the angular projection error of the measured velocities, as the maximum error given the plate dimensions and the distance between the laser and the structure was of approximately six percent.

The plate was excited with a sinusoidal force at $128 \mathrm{~Hz}$ and the magnitude and phase of the transverse vibration velocities over its surface were measured relative to the force transducer signal with the scanning LDV. Figure 10 shows the measured mobility ODS smoothed once using a median filter to remove outliers (noise "spikes") and the surface approximated using a two-dimensional RDFS with $p=q=4$, $\mathrm{M} / M=\mathrm{N} / N=1.5$. A very good agreement with the corresponding ODS predicted with the FE model can be observed (see Fig. 4). The correlation coefficient between the measured and predicted ODS's is 0.95, although no model updating procedure was used to try to match the FE predictions to the measurements.

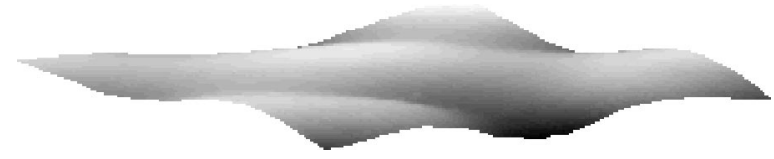

(a)

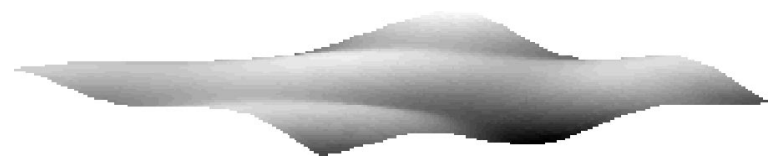

(b)

Fig. 10. Mobility ODS at $128 \mathrm{~Hz}$. (a) measured; (b) approximated using a 2-D RDFS with $p=q=4, \mathrm{M} / M=\mathrm{N} / N=1.5$.

Figure 11 shows the power flow computed from the measured ODS using different methods. Finally, Fig. 12 shows the divergence plots computed from the power flow fields of the previous figure.

The power flow patterns in Fig. 11 are very similar to the power flow patterns computed for the simulated results, shown previously in Figs 5 and 6. The power flow including near-field and wave interaction effects, in Fig. 11(a) is similar to the power flow maps 


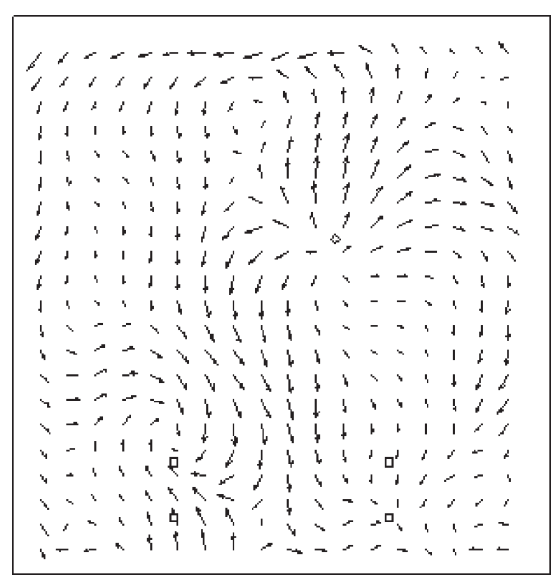

(a)

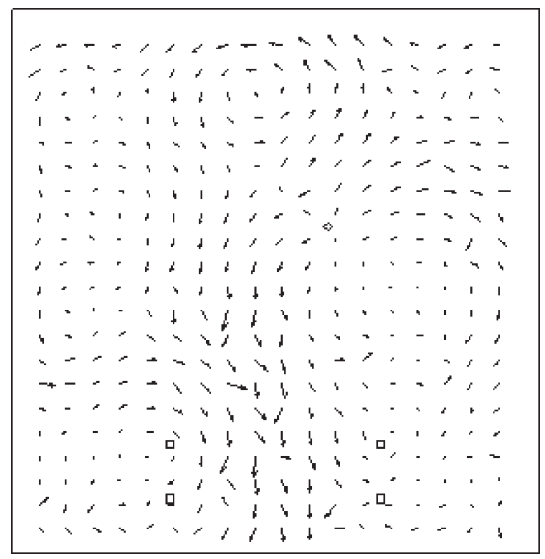

(c)

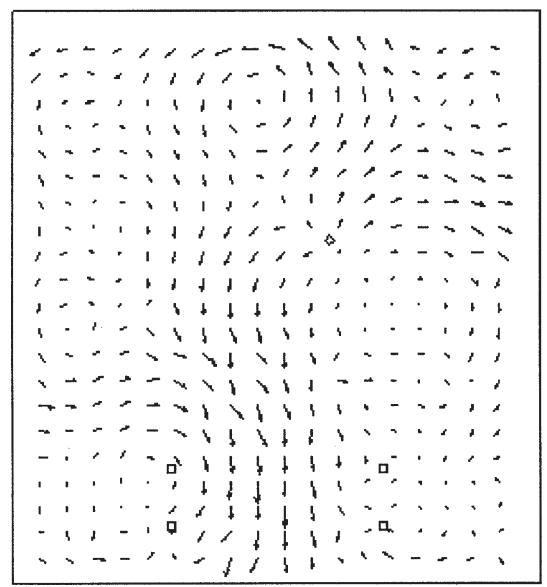

(e)

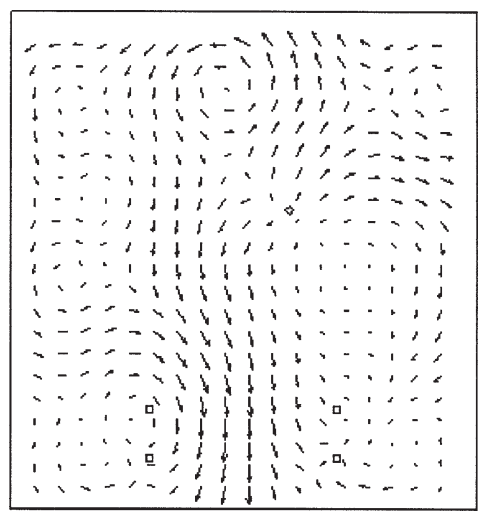

(b)

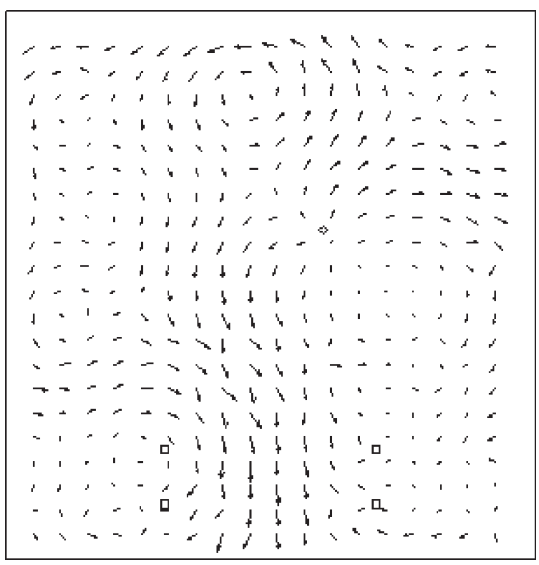

(d)

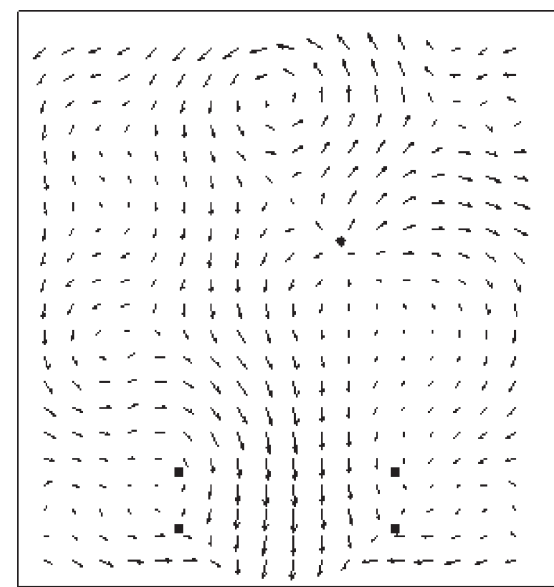

(f)

Fig. 11. Structural power flow at $128 \mathrm{~Hz}$ computed from the measured ODS of Fig. 10 using the RDFS approximation with (a) Eq. (21) and (b) Eq. (22); finite differences using (c) Eq. (27); (d) Eq. (28); (e) Eq. (29); (f) Eqs (30a)-(30c). 


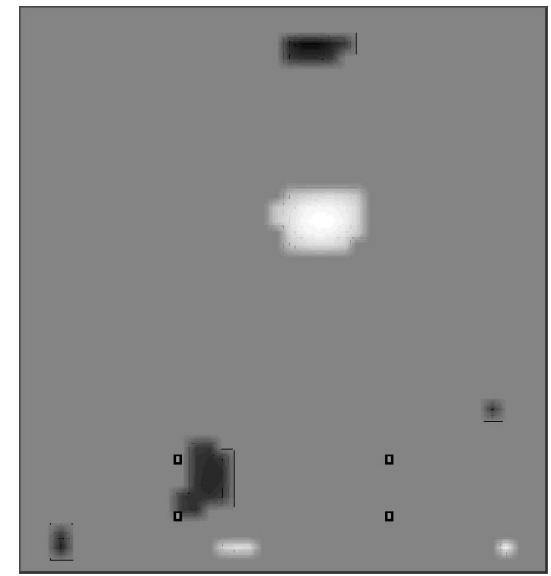

(a)

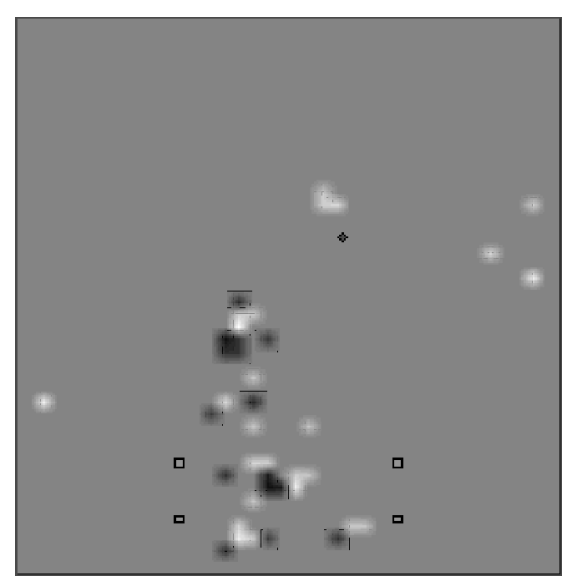

(c)

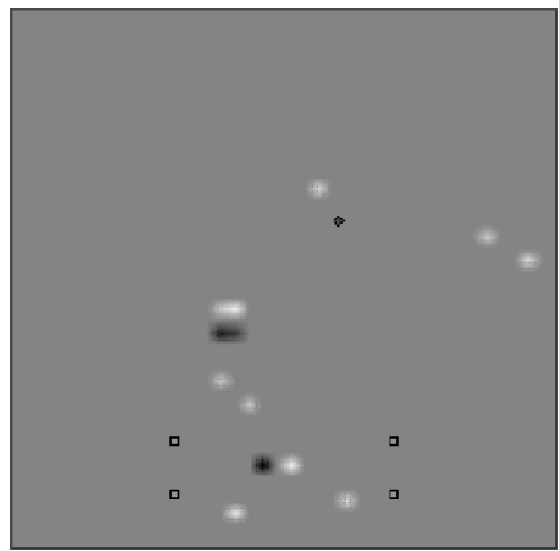

(e)

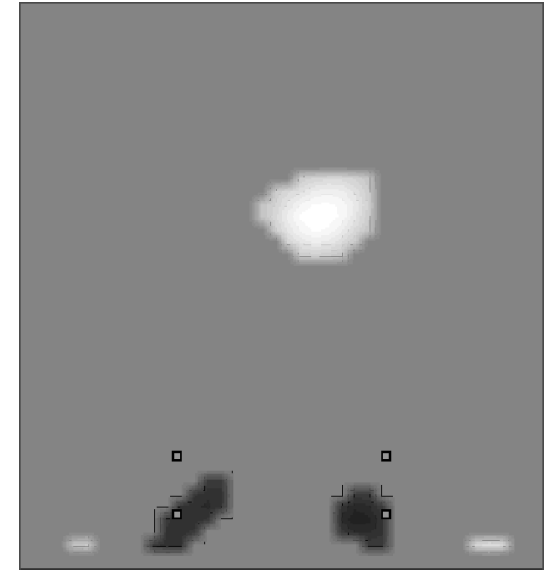

(b)

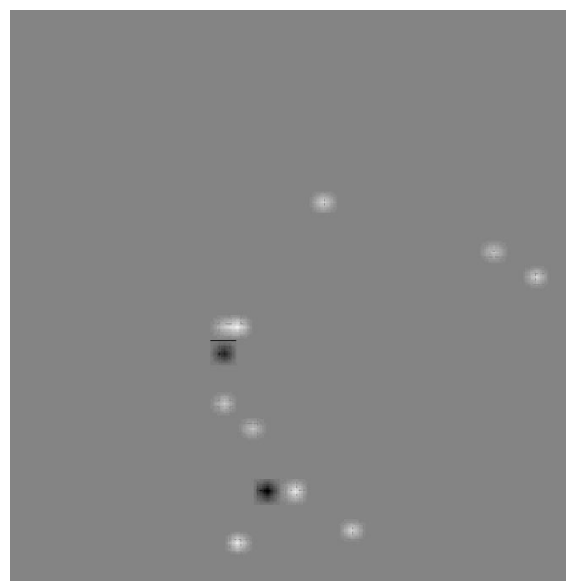

(d)

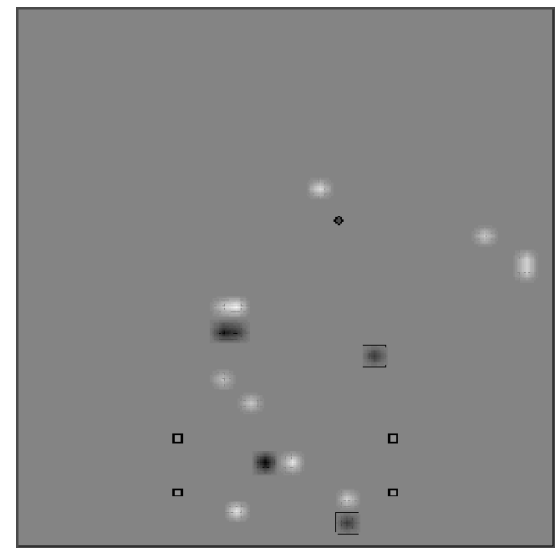

(f)

Fig. 12. Divergence plots computed from the power flow fields shown in Fig. 11. RDFS approximation with (a) Eq. (21) and (b) Eq. (22); finite differences using (c) Eq. (27); (d) Eq. (28); (e) Eq. (29); (f) Eqs (30a)-(30c). 
of Fig. 5(a) and Fig. 6(a). The difference with respect to the power flow computed assuming free-field propagation can be observed mainly in the region in between the rubber mounts. The finite difference approximations produce power flow patterns which are similar to the power flow computed with the RDFS under the far field assumption. The more elaborate finite difference schemes using 5 accelerations and 10 cross spectra and using 9 accelerations and 20 cross spectra yielded better results (see Figs 11(e)-(f)), probably not because they involve higher order derivatives, but because of the averaging effect of using more data to compute the power flow.

Although the power flow fields computed using the finite difference schemes seem qualitatively reasonable, the divergence plots computed from them are completely meaningless, as it can be seen in Figs 12(c)-(f). The errors in the power flow amplitudes cause large errors in the divergence computation. Only the divergence plots computed using the RDFS allow the localization of the source and sinks, especially the results obtained using the free-field assumption. This was expected, as similar behavior was observed with simulated data, i.e., the errors in the higher order derivatives are such that including them in the computation of the power flow is more detrimental than beneficial.

\section{Conclusions}

Structural power flow measurements are difficult because the active part of the total vibration energy is usually very small. The information about the active power flow is in the spatial derivatives of the measured vibration field, and it is known that differentiation causes noise amplification. In the case of flexural power flow in thin plates, using the free-field assumption allows the determination of the power flow using only the first spatial derivative. Otherwise, to take into account near-field effects, spatial derivatives up to the third order are necessary.

A technique was proposed that takes advantage of the spatially dense vibration measurements obtained using scanning laser vibrometers. The measured operational deflection shapes are approximated using a two-dimensional discrete Fourier series with arbitrary fundamental period (not an interpolation like the DFT). The arbitrary period minimizes leakage and allows a dramatic low pass filtering of the data while preserving its spatial information. From the obtained wavenumber-frequency data it is straightforward to compute the flexural power flow in plates.

The formulation of the problem of flexural power flow in thin plates including finite difference implementations was reviewed. The proposed method was appraised and compared with existing finite difference schemes to compute the structural power flow.

It was shown that, although the technique allows including near-field effects when computing the power flow, the results obtained using the free-field assumption were better, both when using simulated data and experimental data. Especially, when a divergence field is to be computed from the intensity field, the free-field assumption becomes essential. Results obtained with the proposed method were better than results obtained using finite difference formulations, mainly when using experimental results.

The method presented is this paper can be used to determine the structural power flow pattern and divergence fields from operational deflection shapes measured with scanning laser vibrometers. The divergence fields can be used to localize energy sources and sinks. Also, by integrating the intensity field along a closed path encircling the power sources and sinks, it is possible to determine the power injected or dissipated.

\section{Acknowledgements}

The author is thankful to Professor Paul Sas, PMA Division, Mechanical Engineering Department, Katholieke Universiteit Leuven for this research opportunity. The financial support of the Brazilian research funding agency CNPq (Proc. No. 200028/86-3) and of the Katholieke Universiteit Leuven are also acknowledged.

\section{References}

[1] J.R.F. Arruda, Surface smoothing and partial derivatives computation using a regressive discrete Fourier series, Mechanical Systems and Signal Processing 6(1) (1992), 41-50.

[2] P.D. Bauman, Measurement of structural intensity: analytic and experimental evaluation of various techniques for the case of flexural waves in one-dimensional structures, J. Sound and Vibration 174(5) (1994), 677-694.

[3] J.D. Blotter and R.L. West, Experimental and analytical energy and power flow using a scanning laser Doppler vibrometer, in: Proc. 1st Int. Conf. Vibration Measurements by Laser Techniques, SPIE Vol. 2358, Ancona, Italy, 1994, pp. 266-275. 
[4] X. Carniel, Mésure de la propagation de l'énergie vibratoire dans les barres, méthodes intensimetriques, Thèse de Doctorat, Université du Maine, France, 1987 (in French).

[5] J.F. Doyle, Wave Propagation in Structures, Springer, New York, 1989.

[6] L. Gavric and G. Pavic, Computations of structural intensity in beam-plate structures by numerical modal analysis using FEM, in: Proc. 3rd Int. Congress on Intensity Techniques, Senlis, France, 1990, pp. 207-214.

[7] B. Meyer and D. Thomasson, L'intensimétrie vibratoire: description, mise en oeuvre, résultats, in: EDF Bulletin de la direction des études et recherches-Série A 3, Suppl. 4, 1989, pp. 69-86 (in French).

[8] D.U. Noiseaux, Measurement of power flow in uniform beams and plates, J. Acoust. Soc. America 47 (1970), 238-247.
[9] J.-C. Pascal, T. Loyau and J.A. Mann III, Structural intensity from spatial Fourier transformation and Bahim acoustical holography method, in: Proc. 3rd Int. Congress on Intensity Techniques, Senlis, France, 1990, pp. 197-204.

[10] J.-C. Pascal, T. Loyau and X. Carniel, Complete determination of structural intensity in plates using laser vibrometers, J. Sound and Vibration 161(3) (1993), 527-531.

[11] G. Pavic, Measurement of structure borne wave intensity, part I: formulation of methods, J. Sound and Vibration 49(2) (1976), 221-230.

[12] J.S. Przemieniecki, Theory of Matrix Structural Analysis, McGraw-Hill, New York, 1968.

[13] J.W. Verheij, Cross-spectral density methods for measuring structure-borne power flow on beams and pipes, J. Sound and Vibration 70 (1980), 133-139. 

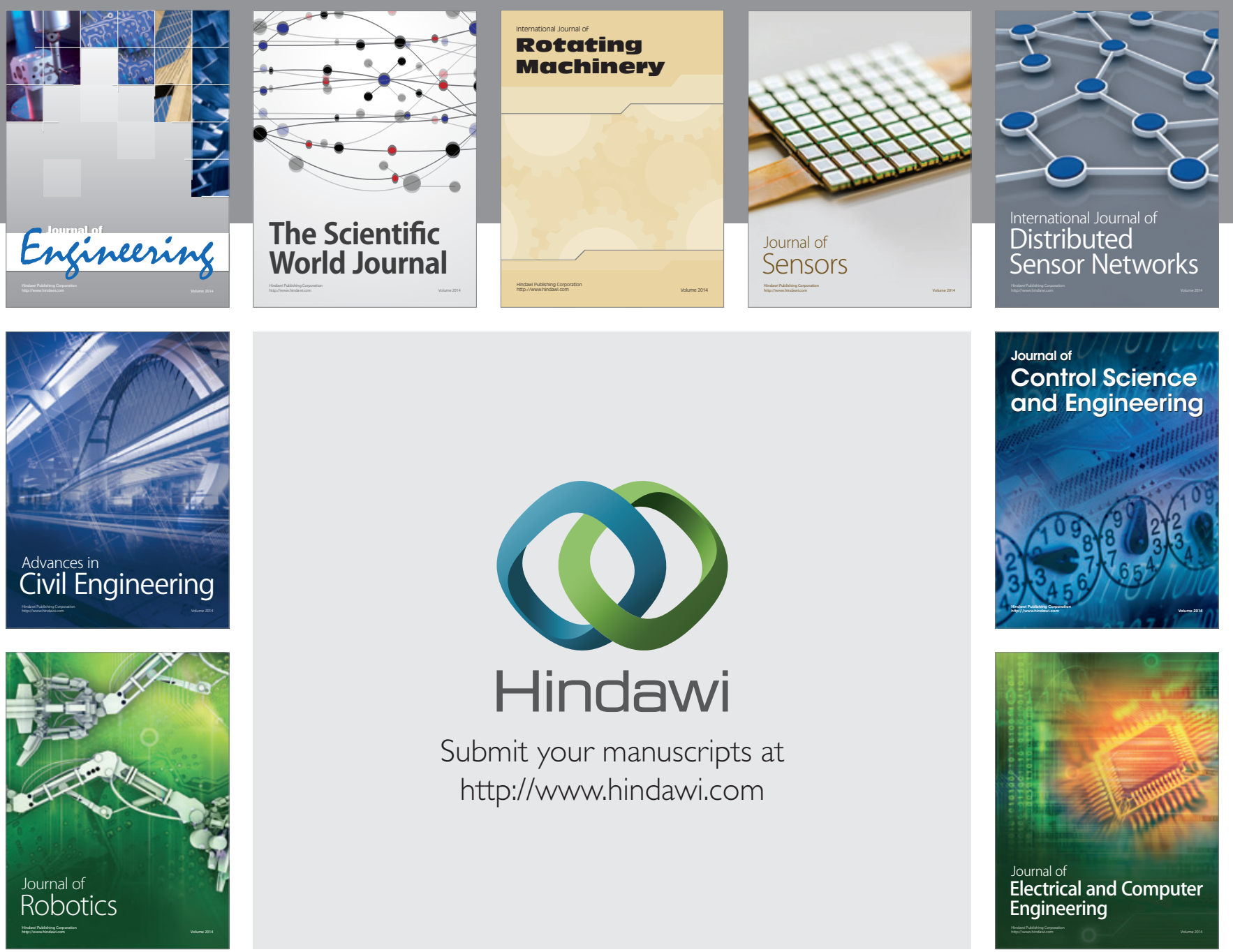

Submit your manuscripts at

http://www.hindawi.com
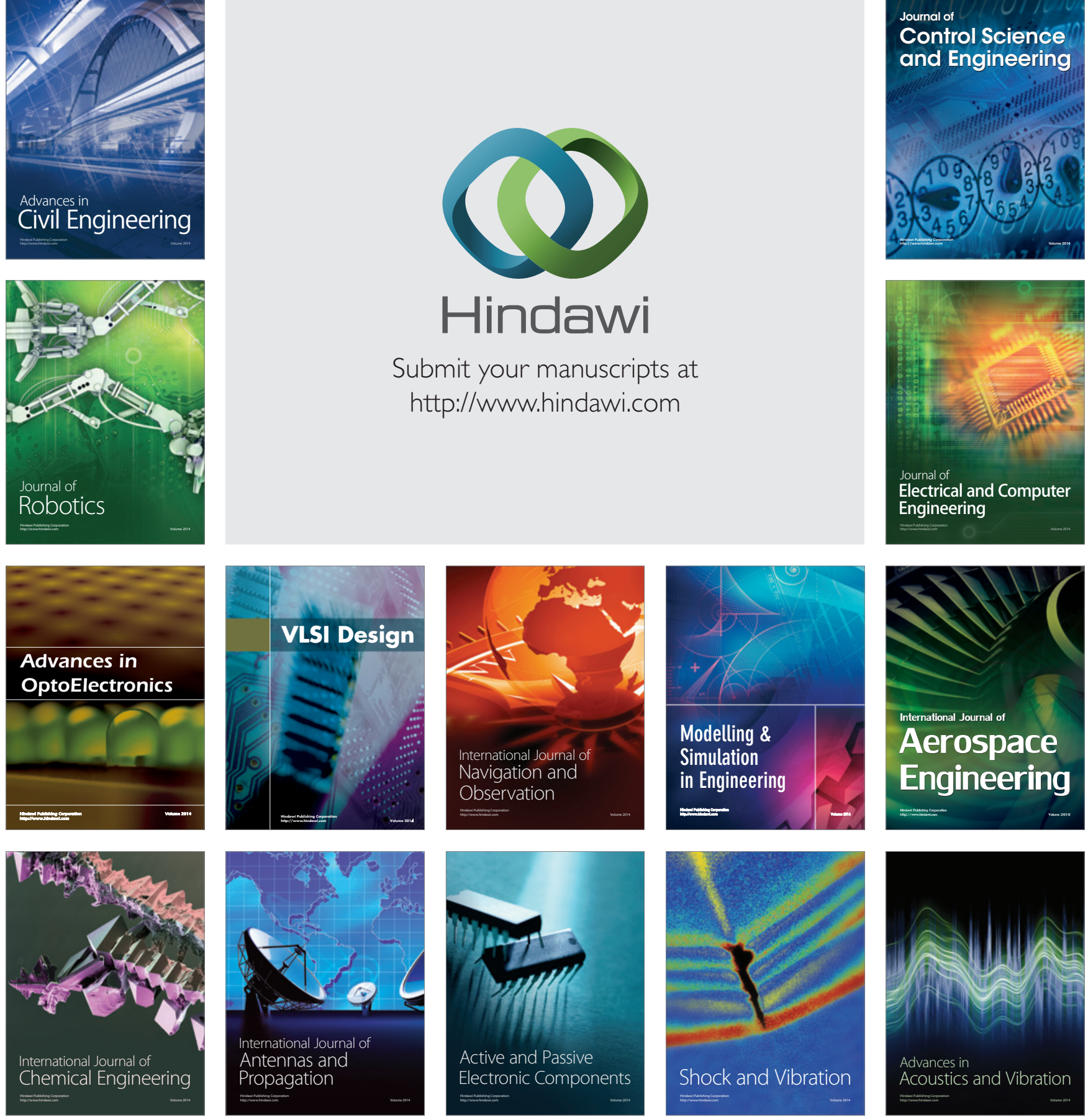